\title{
Non-LTE line formation for heavy elements in four very metal-poor stars ${ }^{\star}$
}

\author{
L. Mashonkina ${ }^{1,2}$, G. Zhao ${ }^{3}$, T. Gehren ${ }^{1}$, W. Aoki ${ }^{4}$, M. Bergemann $^{1}$, \\ K. Noguchi ${ }^{4}$, J. R. Shi ${ }^{3}$, M. Takada-Hidai ${ }^{5}$, and H. W. Zhang ${ }^{6}$ \\ 1 Institut für Astronomie und Astrophysik der Universität München, Scheinerstr. 1, 81679 München, Germany \\ e-mail: lyuda@usm.lmu.de \\ 2 Institute of Astronomy, Russian Academy of Science, Pyatnitskaya 48, 119017 Moscow, Russia \\ e-mail: lima@inasan.ru \\ 3 National Astronomical Observatories, Chinese Academy of Sciences, A20 Datun Road, Chaoyang District, Beijing 100012, \\ PR China \\ 4 National Astronomical Observatory, Mitaka, Tokyo 181-8588, Japan \\ 5 Liberal Arts Education Center, Tokai University, Hiratsuka, Kanagawa 259-1292, Japan \\ ${ }^{6}$ Department of Astronomy, School of Physics, Peking University, Beijing 100871, PR China
}

Received 11 June 2007 / Accepted 16 November 2007

\section{ABSTRACT}

\begin{abstract}
Aims. Stellar parameters and abundances of $\mathrm{Na}, \mathrm{Mg}, \mathrm{Al}, \mathrm{K}, \mathrm{Ca}, \mathrm{Sr}, \mathrm{Ba}$, and $\mathrm{Eu}$ are determined for four very metal-poor (VMP) stars $(-2.15 \geq[\mathrm{Fe} / \mathrm{H}] \geq-2.66)$. For two of them, HD 84937 and HD 122563, the fraction of the odd isotopes of Ba derived for the first time.

Methods. Determination of an effective temperature, surface gravity, and element abundances was based on non-local thermodynamic equilibrium (non-LTE) line formation and analysis of high-resolution $(R \sim 60000$ and 90000$)$ high signal-to-noise $(S / N \geq 200)$ observed spectra. A model atom for $\mathrm{H} \mathrm{I}$ is presented. An effective temperature was obtained from the Balmer $\mathrm{H}_{\alpha}$ and $\mathrm{H}_{\beta}$ line wing fits. The surface gravity was calculated from the HIPPARCos parallax if available and the non-LTE ionization balance between Ca I and Ca II. Based on the hyperfine structure affecting the Ba II resonance line $\lambda 4554$, the fractional abundance of the odd isotopes of $\mathrm{Ba}$ was derived from a requirement that $\mathrm{Ba}$ abundances from the resonance line and subordinate lines of Ba II must be equal.

Results. For each star, non-LTE leads to a consistency of $T_{\text {eff }}$ from two Balmer lines and to a higher temperature compared to the LTE case, by up to $60 \mathrm{~K}$. Non-LTE effects are important in spectroscopic determination of surface gravity from the ionization balance between Ca I and Ca II. For each star with a known trigonometric surface gravity, non-LTE abundances from the lines of two ionization stages, Ca I and Ca II, agree within the error bars, while a difference in the LTE abundances consists of 0.23 dex to 0.40 dex for different stars. Departures from LTE are found to be significant for all investigated atoms, and they strongly depend on stellar parameters. For HD 84937, the $\mathrm{Eu} / \mathrm{Ba}$ ratio is consistent with the relative solar system $r$-process abundances, and the fraction of the odd isotopes of $\mathrm{Ba}, f_{\text {odd }}$, equals $0.43 \pm 0.14$. The latter can serve as an observational constraint on $r$-process models. The lower Eu/Ba ratio and $f_{\text {odd }}=0.22 \pm 0.15$ found for HD 122563 suggest that the $s$-process or the unknown process has contributed significantly to the $\mathrm{Ba}$ abundance in this star.
\end{abstract}

Key words. line: formation - line: profiles - stars: abundances - stars: fundamental parameters - stars: late-type - stars: general

\section{Introduction}

Very metal-poor stars (hereafter VMP stars, with $[\mathrm{Fe} / \mathrm{H}]^{1}<-2$ ) belonging to the halo population of the Galaxy were formed at the earliest epoch of star formation. These stars preserve in the atmosphere the chemical composition produced by the early generation of stars. Determination of element abundances in VMP stars is important for understanding nucleosynthesis processes in the early Galaxy. The more metal-poor stars are, on average, more distant, and they are more difficult to analyze than nearby stars with metal abundances close to solar. In particular, the methods of surface gravity determination are of limited usefulness. For distant field halo stars, trigonometric parallaxes are unknown. Progress in this direction is expected due to the

\footnotetext{
* Based on observations collected at Subaru Telescope, which is operated by the National Astronomical Observatory of Japan.

${ }^{1}[\mathrm{~A} / \mathrm{B}]=\log \left(N_{A} / N_{B}\right)_{*}-\log \left(N_{A} / N_{B}\right)_{\odot}$ where $N_{X}$ are number densities.
}

upcoming ESA Gaia satellite mission (Perryman et al. 2001). The strongest lines of neutral magnesium (the $\mathrm{Mg} \mathrm{Ib}$ lines) and neutral calcium (the $\mathrm{Ca}$ I triplet $4 \mathrm{p}-5 s$ ) show no wings, and, therefore, they are not sensitive to surface gravity. In fact, surface gravities of VMP stars can only be found from the ionization balance between neutral and ionized species of a selected atom. One mostly selects iron and Fe peak elements. In two recently found hyper metal-poor stars (hereafter HMP stars, with $[\mathrm{Fe} / \mathrm{H}]<-5$ ), only $\mathrm{Ca}$ is observed in two ionization stages (Christlieb et al. 2002; Frebel et al. 2005) and, therefore, can be a potential tool for the derivation of $\log g$.

The atmospheres of VMP stars are characterized by low electron number densities and low opacities, which are particularly low in the ultraviolet spectral range. Hence, local thermodynamical equilibrium (LTE) is not fulfilled in the atmospheric layers where the lines are formed. As was proven in the past decade, the surface gravity based on the ionization balance $\mathrm{Fe} / \mathrm{Fe}$ II 
(Thevenin \& Idiart 1999; Korn et al. 2003) and element abundances (for review, see Asplund 2005) are significantly affected by departures from LTE. In this study, we select four metalpoor stars observed with the High Dispersion Spectrograph of the Subaru Telescope and perform detailed analysis of stellar parameters and element abundances based on the non-local thermodynamic equilibrium (non-LTE) line formation for 10 important chemical species, including H I, Ca I, Ca II, etc. One of the stars, HD 122563, is a giant, and the remaining three, HD 84937, $\mathrm{BD}+3^{\circ} 740$, and $\mathrm{BD}-13^{\circ} 3442$, are at the hot end of the stars that evolve on time scales comparable to the Galaxy lifetime. Similar intrinsically bright objects are mainly found among VMP stars due to the selection effect. For example, one of two HMP stars, the Christlieb's star is a giant with $\log g=2.2$ (Christlieb et al. 2004) and another one has an effective temperature $T_{\text {eff }}=6180 \mathrm{~K}$ (Aoki et al. 2006). In the selected stars, it is possible to study the formation of some of the strongest spectral lines, such as Ca II $\lambda 8498, \mathrm{Mg}$ I $\lambda 5172$ and $\lambda 5183$, which are among the few spectral lines observed in HMP stars. At $[\mathrm{Fe} / \mathrm{H}]<-2$, they are presumably of purely photospheric origin. Thus, the stars of our small sample can serve as reference stars in studies of VMP and HMP stars.

The paper is organized as follows. Observations of the selected stars are described in Sect. 2. Non-LTE calculations are found in Sect. 3. The solar element abundances necessary for further differential abundance analysis are derived in Sect. 4 from the selected spectral lines. In Sect.5, we determine effective temperatures from the wings of Balmer lines and check surface gravities from the ionization balance $\mathrm{Ca}$ I/Ca II. Non-LTE abundances of 8 chemical elements are derived in Sect. 6. For two stars, HD 84937 and HD 122563, both the Ba II resonance and subordinate lines are available, and we determine in Sect. 7.2 the fraction of the odd isotopes of barium that gives an independent estimate of relative contribution of the $r$-process and main $s$-process to heavy element abundances in the star. Our recommendations and conclusions are given in Sect. 8.

\section{Observations and data reductions}

The spectroscopic observations for our program stars were carried out on Feb. 2, 2002 with the High Dispersion Spectrograph (HDS; Noguchi et al. 2002) at the Nasmyth focus of the Subaru $8.2 \mathrm{~m}$ telescope. Signal-to-noise $(S / N)$ ratio measured per pixel $\left(0.9 \mathrm{~km} \mathrm{~s}^{-1}\right)$ is 200 to 400 for the brightest star HD 122563 and is lower, down to 100, for the remaining three stars. We use also high-quality observed spectra covering Ca II 3933, Ca II 8498, the lines of Al I and K I from the ESO UVESPOP survey (Bagnulo et al. 2005) for HD 84937 and HD 122583 and from the ESO/UVES archive (Program ID: 67.D-0106 by Nissen; 67.D-0439 and 68.D-0094 by Primas) for $\mathrm{BD}+3^{\circ} 740$ and $\mathrm{BD}-13^{\circ} 3442$. The Balmer lines $\mathrm{H}_{\alpha}$ and $\mathrm{H}_{\beta}$ in HD 84937 and $\mathrm{BD}+3^{\circ} 740$ are studied using observational data obtained by Klaus Fuhrmann with the fiber optics Cassegrain echelle spectrograph FOCES at the $2.2 \mathrm{~m}$ telescope of the Calar Alto Observatory in 1999 and 1995. The high quality of the FOCES data reduction accuracy has been emphasized by Korn (2002) who presents evidence for his claim that systematic errors of the effective temperatures derived from the Balmer line wing fits due to data reduction (rectification and flatfielding) are below $50 \mathrm{~K}$. This holds for HD 84937 . For the BD $+3^{\circ} 740 \mathrm{spec}-$ trum, the error may reach a slightly higher value $(\sim 70 \mathrm{~K})$, because before 1997 all spectra were degraded by mode transfer noise in the optical fibers (Grupp 2003) that did not allow an
$S / N>150-200$. Characteristics of observed spectra are summarized in Table 1.

The Subaru and ESO/UVES spectra were reduced with a standard MIDAS/ECHELLE package for order identification, background subtraction, flat-fielding, order extraction, and wavelength calibration. Bias, dark current, and scattered light correction are included in the background subtraction. The spectrum was then normalized by a continuum function determined by fitting a spline curve to a set of pre-selected continuum windows estimated from the Kitt Peak Solar Atlas (Kurucz et al. 1984).

\section{Non-LTE calculations}

All the investigated elements are assumed to be trace elements, which means that we obtain statistical equilibrium (SE) populations for each of them while keeping the atmospheric structure fixed. In the stellar parameter range we are concerned with, such approach is justified both for metals and for hydrogen. Hydrogen is not an important continuous opacity source compared to the $\mathrm{H}^{-}$ions at temperature below $7000 \mathrm{~K}$. Although it becomes a dominant electron donor in the atmospheres of metal-poor stars $([\mathrm{Fe} / \mathrm{H}]<-1)$, our non-LTE calculations show that the ionization balance between $\mathrm{HII}$ and $\mathrm{HI}$ is close to thermodynamic equilibrium (TE) in the layers where the Balmer line wings form. For instance, the population ratio $\mathrm{H} \mathrm{II} / \mathrm{H} \mathrm{I}$ deviates by no more than $1 \%$ from the TE value below $\log \tau_{5000}=-2.5$ in the model with $T_{\text {eff }}=6390 \mathrm{~K}, \log g=3.88$, and $[\mathrm{Fe} / \mathrm{H}]=-2.66$. Based on the self-consistent non-LTE modelling of a solar-type star, Short $\&$ Hauschildt (2005) conclude that the non-LTE effects of all the light metals (including $\mathrm{Na}, \mathrm{Mg}, \mathrm{Al}, \mathrm{K}$, and $\mathrm{Ca}$ among others) on the model structure and flux distribution are small.

Calculations are performed with the plane-parallel, homogeneous, LTE, and blanketed model atmospheres computed for individual stellar parameters using the code MAFAGS (Fuhrmann et al. 1997). For the program metal-poor stars, $\alpha$-enhancement is accounted for with the abundances of $\mathrm{Mg}$ and $\mathrm{Ca}$ determined in this study, the $\mathrm{Si}$ abundance that follows the $\mathrm{Mg}$ one, and the oxygen abundance taken from the literature. We assume $[\mathrm{O} / \mathrm{Fe}]=0.5$ where no other data is available. It is worth noting that oxygen in cool stellar atmospheres plays a minor role as a donator of free electrons and as opacity source, and the uncertainty of its abundance does not affect the calculated atmospheric structure.

In order to solve the coupled radiative transfer and statistical equilibrium equations for metals, we use a revised version of the DETAIL program (Butler \& Giddings 1985) based on the accelerated lambda iteration, which follows the efficient method described by Rybicki \& Hummer (1991, 1992). NonLTE computations for H I are based on the complete linearization method as described by Auer \& Heasley (1976). We apply the code NONLTE3 originally treated by Sakhibullin (1983) and advanced later (Kamp et al. 2003). The departure coefficients are then used to compute the synthetic line profiles. The metal line list has been extracted from Kurucz' (1992) compilation.

\subsection{Non-LTE line formation for $\mathrm{HI}$}

\subsubsection{Model atom of $\mathrm{HI}$}

We consider non-LTE line formation for H I in order to provide diagnostics for the Balmer lines $\mathrm{H}_{\alpha}$ and $\mathrm{H}_{\beta}$ that are used for an accurate temperature determination in cool stars. The model atom of $\mathrm{HI}$ includes levels with principal quantum 
Table 1. Characteristics of observed spectra.

\begin{tabular}{llcll}
\hline \hline $\begin{array}{l}\text { Telescope/ } \\
\text { spectrograph }\end{array}$ & \multicolumn{1}{c}{ Spectral range $(\AA)$} & $R$ & $S / N$ & Objects \\
\hline Subaru/HDS & $4120-5430,5520-6860$ & 90000 & $\geq 200$ & HD 122563 \\
& & & $\geq 100$ & HD 84937, BD $+3^{\circ} 740, \mathrm{BD}-13^{\circ} 3442$ \\
VLT2/UVES & $3850-8550$ & 80000 & $\geq 200$ & HD 122563, HD 84 937 \\
VLT2/UVES & $3730-4990,4760-6840$, & 48000, & $\geq 200$ & BD $+3^{\circ} 740, \mathrm{BD}-13^{\circ} 3442$ \\
& $6600-10600$ & 60000 & & \\
$2.2-m / F O C E S$ & $4500-6700$ & 60000 & $\geq 100$ & HD 84 937 \\
& & 35000 & $\geq 100$ & $\mathrm{BD}+3^{\circ} 740$ \\
\hline
\end{tabular}

numbers up to $n \leq 19$ and energies adopted from Wiese et al. (1966). Transition probabilities are taken from the Vienna Atomic Line Data base (Kupka et al. 1999) and if not available they are computed using the approximate formula of Bethe \& Salpeter (1957) as implemented by Johnson (1972). Photoionization cross-sections are evaluated using the exact expression for the hydrogen atom. Collisional rates include contributions from inelastic collisions with electrons and hydrogen atoms. For electron-impact excitation, the $R$-matrix calculations of Przybilla \& Butler (2004a) are used for the transitions between the energy levels with $n \leq 7$ and the approximation formula of Johnson (1972) for the remainder. For electron-impact ionization, we apply the Seaton formula as described by Mihalas (1978). The data on inelastic collisions with neutral H particles available in literature for $\mathrm{H}$ I have been recently investigated by Barklem (2007). He concludes that they all are of questionable quality. Here we use the Drawin's (1968) formula as implemented by Steenbock \& Holweger (1984). Since it provides only an order of magnitude estimate, we constrain the efficiency of hydrogenic collisions empirically. The rates calculated using this formula were multiplied by a scaling factor $S_{\mathrm{H}}=0$ (no hydrogenic collisions), $0.1,1$, and 2 in order to make the effective temperatures derived from the two Balmer lines $\mathrm{H}_{\alpha}$ and $\mathrm{H}_{\beta}$ in the selected stars consistent. Our best estimate $S_{\mathrm{H}}=2$ agrees with that of Przybilla \& Butler (2004b).

Radiation transfer is treated explicitly for all Balmer and Paschen lines, for the first seven Lyman lines, for every boundbound transition with $n_{\text {low }}=4-8$ and $n_{\text {up }} \leq 13$, and for the four continua including Lyman, Balmer, Paschen and Brackett ones, in total, for 70 transitions. Radiative rates for the remaining transitions are evaluated using LTE mean intensities obtained from the formal solution of the radiation transfer equation. For the transitions arising between the energy levels with $n \leq 8$, the line absorption profile is computed in detail and includes Stark broadening, radiative damping, self-broadening, Doppler broadening (both thermal and turbulent), and fine structure as implemented by Barklem \& Piskunov (2003) in their subroutine HLINOP. For the remaining transitions, Doppler profiles are assumed.

\subsubsection{Departures from LTE for $\mathrm{H}$ I}

Our calculations show that the behavior of the departure coefficients $b_{i}=n_{i}^{\mathrm{NLTE}} / n_{i}^{\mathrm{LTE}}$ and the line source functions are similar for all the models representing the atmospheres of the Sun and selected stars. Here, $n_{i}^{\text {NLTE }}$ and $n_{i}^{\text {LTE }}$ are the statistical equilibrium and thermal (Saha-Boltzmann) number densities, respectively. In Fig. 1, the departure coefficients of the lowest 7 levels of $\mathrm{HI}$ are shown as a function of continuum optical depth $\tau_{5000}$ referring to $\lambda=5000 \AA$ in the model atmosphere of $\mathrm{BD}+3^{\circ} 740$.

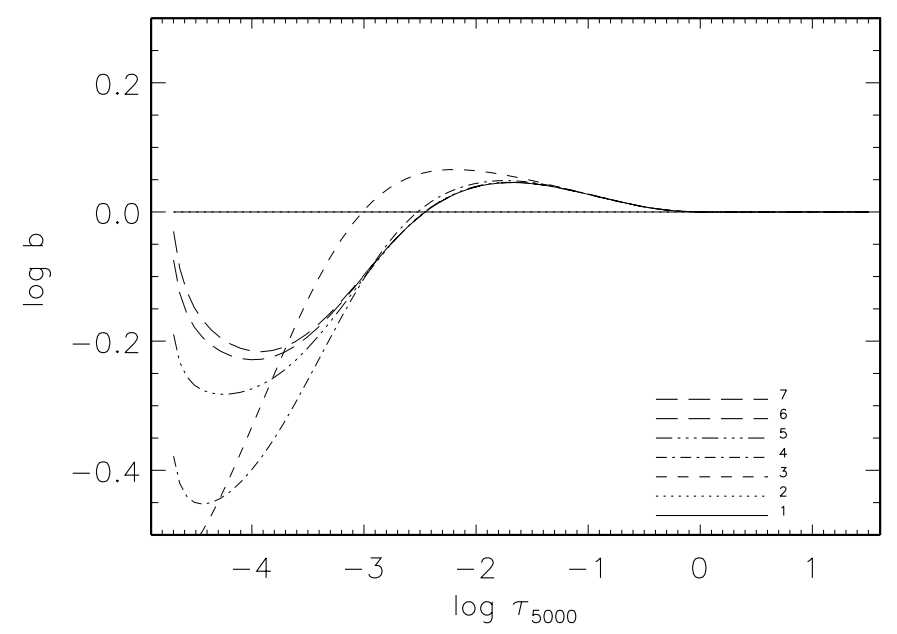

Fig. 1. Departure coefficients $\log b_{i}$ for the first seven levels of $\mathrm{HI}_{\mathrm{I}}$ in the model atmosphere of $\mathrm{BD}+3^{\circ} 740$.

The ground state and the first excited level $(n=2)$ keep their TE level populations throughout the atmosphere. Departures from LTE for the $n=3$ level are controlled by $\mathrm{H}_{\alpha}$. In the layers, where the continuum optical depth drops below unity, $\mathrm{H}_{\alpha}$ serves as the pumping transition resulting in a slight overpopulation (up to $\simeq 5 \%$ ) of the upper level. But starting from $\log \tau_{5000} \simeq-3$ and further out, photon escape from $\mathrm{H}_{\alpha}$ wings causes an underpopulation of the $n=3$ level. The levels with $n \geq 4$ stay in nearly detailed balance relative to each other.

For the Balmer lines of particular interest, $\mathrm{H}_{\alpha}$ and $\mathrm{H}_{\beta}$, nonLTE leads to slight weakening of the core-to-wing transition compared to the LTE case and significant strengthening of the core. The core-to-wing transition is of particular importance for temperature determinations, because this part of profile is sensitive to $T_{\text {eff }}$ variations.

We find that classical theoretical model atmospheres fail to reproduce a half-width of the $\mathrm{H}_{\alpha}$ and $\mathrm{H}_{\beta}$ lines in the Sun (Fig. 2 for $\mathrm{H}_{\alpha}$ ) and cool stars (Figs. 3 and 5). Similar conclusion is drawn by Przybilla \& Butler (2004b), Fuhrmeister et al. (2006); for $\mathrm{H}_{\alpha}$ in the Sun. Therefore the line core within $0.7 \AA$ to $1.5 \AA$, with the exact number depending on stellar parameters, is not included in the fit. In the entire range of stellar parameters we are concerned with, we obtain weak non-LTE effects for the $\mathrm{H}_{\beta}$ profile beyond the core independent of the applied scaling factor $S_{\mathrm{H}}$. For example, for the model representing the atmosphere of $\mathrm{BD}+3^{\circ} 740\left(T_{\mathrm{eff}}=6340 \mathrm{~K}, \log g=3.90,[\mathrm{Fe} / \mathrm{H}]=-2.65\right)$, a difference in the LTE and non-LTE $\left(S_{\mathrm{H}}=0.1\right)$ profiles equals $0.1 \%$ of the continuum flux at $\Delta \lambda=1.7 \AA$ from the line center and rapidly decreases with increasing $\Delta \lambda$. In fact, non-LTE effects can be neglected deriving the effective temperature from $\mathrm{H}_{\beta}$. For 


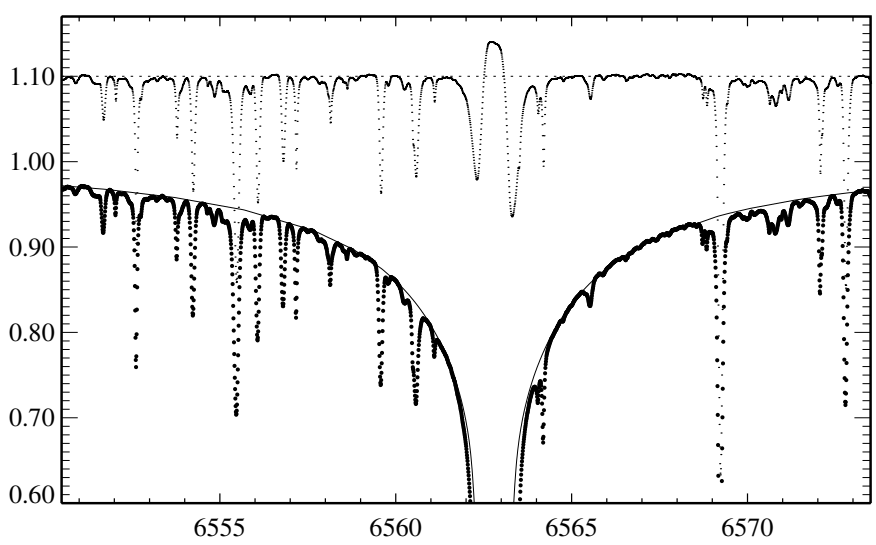

Fig. 2. Theoretical non-LTE flux profile (continuous line) of the Balmer $\mathrm{H}_{\alpha}$ line computed for $T_{\text {eff }}=5780 \mathrm{~K}$ using the $A G$ self-broadening recipe compared to the observed spectrum of the Kurucz et al. (1984) solar flux atlas (bold dots). The differences between observed and calculated spectra $(\mathrm{O}-\mathrm{C})$ are presented in the upper part of figure.

$\mathrm{H}_{\alpha}$, the corresponding difference equals $2.7 \%$ of the continuum flux at $\Delta \lambda=1.7 \AA$ and becomes smaller than $0.1 \%$ only beyond $\Delta \lambda=6.7 \AA$. A difference in the non-LTE $\mathrm{H}_{\alpha}$ profiles computed with $S_{\mathrm{H}}=1$ and 2 is smaller than $0.1 \%$ of the continuum flux everywhere beyond $\Delta \lambda=1.7 \AA$. Both profiles are $0.7 \%$ stronger compared to that computed with $S_{\mathrm{H}}=0.1$ at $\Delta \lambda=1.7 \AA$. The three profiles agree within $0.1 \%$ beyond $\Delta \lambda=3.2 \AA$. In Sect. 5.3, we inspect an influence of the $S_{\mathrm{H}}$ variation on the derived $T_{\mathrm{eff}}$ of $\mathrm{BD}+3^{\circ} 740$. Similar effects are expected for other program stars.

\subsection{3. $\mathrm{H}_{\alpha}$ in the solar spectrum}

Hereafter, the theoretical profiles of $\mathrm{H}_{\alpha}$ and $\mathrm{H}_{\beta}$ are computed as accurately as possible, including a convolution of the profiles resulting from the different broadening mechanisms. The Stark broadening profiles from Vidal et al. (1970, 1973) are employed. For self-broadening, we use two recipes. The first one is based on the Ali \& Griem (1966) theory (hereafter $A G$ ) and the second one on the self-broadening formalism of Barklem et al. (2000, hereafter $B P O$ ). We apply the revised subroutine HYDLINE incorporated in the SIU code.

Non-LTE modelling of $\mathrm{H}_{\alpha}$ has been tested by comparing with solar flux observations taken from the Kitt Peak Solar Atlas (Kurucz et al. 1984). We use the grid of the MAFAGS model atmospheres with various $T_{\text {eff }}$ but with the fixed values of $\log g=$ 4.44 and solar metal abundance. The line core within $\pm 1.1 \AA$ is not included in the fit. We obtain smaller non-LTE effects for $\mathrm{H}_{\alpha}$ in the Sun compared to that found in our program metal-poor stars. The best non-LTE fit is achieved for $T_{\text {eff }}(A G)=5780 \mathrm{~K}$ when the $A G$ recipe is applied (Fig. 2) and for a lower temperature $T_{\text {eff }}(B P O)=5720 \mathrm{~K}$ in the case of the $B P O$ recipe. In each case, the LTE calculated wings for the same temperature are slightly deeper (by up to $0.3 \%$ ) than the observed profile, indicating a $30 \mathrm{~K}$ lower effective temperature value. The $\mathrm{H}_{\beta}$ wings are strongly blended in the solar spectrum. They are satisfactorily fitted using $T_{\mathrm{eff}}(A G)=5780 \mathrm{~K}$.

\subsection{Non-LTE calculations for metals}

For metals, our present investigation is based on the non-LTE methods treated in our earlier studies and documented in a number of papers, where atomic data and the problems of line
Table 2. Atomic models used in this study where NL is the number of levels in the included species ${ }^{1}$.

\begin{tabular}{|c|c|c|c|}
\hline Atom & & NL & Reference \\
\hline Sodium & $\overline{\mathrm{NaI}}$ & 58 & $\begin{array}{l}\text { Baumüller et al. (1998), } \\
\text { Gehren et al. (2004), } \\
\text { Shi et al. (2004) }\end{array}$ \\
\hline Magnesium & $\operatorname{Mg} \mathbf{I}$ & 85 & $\begin{array}{l}\text { Zhao et al. (1998), } \\
\text { Gehren et al. (2004) }\end{array}$ \\
\hline Aluminium & $\mathrm{Al} \mathrm{I}$ & 59 & Baumüller et al. (1996) \\
\hline Potassium & K I & 67 & Zhang et al. (2006a, 2006b) \\
\hline Calcium & $\begin{array}{l}\mathrm{Ca} \text { I } \\
\mathrm{Ca} \text { II }\end{array}$ & $\begin{array}{l}63 \\
37\end{array}$ & Mashonkina et al. (2007a) \\
\hline Strontium & Sr II & 40 & $\begin{array}{l}\text { Belyakova \& Mashonkina (1997), } \\
\text { Mashonkina \& Gehren (2001) }\end{array}$ \\
\hline Barium & Ba II & 35 & Mashonkina et al. (1999) \\
\hline Europium & $\mathrm{Eu}$ II & $63^{2}$ & $\begin{array}{l}\text { Mashonkina (2000), } \\
\text { Mashonkina \& Gehren (2000), } \\
\text { Mashonkina \& Vinogradova (2007) }\end{array}$ \\
\hline
\end{tabular}

${ }^{1}$ Each model atom is closed with the ground state of the next ionization stage, ${ }^{2}$ combined levels based on 176 energy levels of Eu II known from laboratory measurements.

formation have been considered in detail. Table 2 lists the investigated atoms and cites the related papers. For Ca II, the advanced effective collision strengths are used from the recent $R$-matrix calculations of Meléndez et al. (2007).

We note that the most accurate atomic data are used in our non-LTE calculations. For minority species such as $\mathrm{Mg}$ I, Al I, and Ca I, where departures from LTE are mainly caused by superthermal radiation of non-local origin below the thresholds of low excitation levels, photoionization cross-sections are from the Opacity Project calculations (Seaton et al. 1994). Their accuracy is estimated at the level of $10 \%$. Such small uncertainty translates to the abundance error of no more than 0.01 dex. For the collision-dominated atoms $\mathrm{Na}$ I and $\mathrm{K} \mathrm{I}$ and the majority species such as Ca II, Sr II, Ba II, and Eu II, the main source of uncertainties is collisional data. In SE calculations, we account for inelastic collisions both with electrons and neutral $\mathrm{H}$ particles. Hydrogen collisions are computed using the formula of Steenbock \& Holweger (1984) with a scaling factor $S_{\mathrm{H}}$ found empirically from their different influence on the different lines of a given atom in solar and stellar spectra. We apply $S_{\mathrm{H}}=0$ (no hydrogenic collisions) for Sr II, Ba II, and Eu II as estimated by Mashonkina \& Gehren $(2000,2001)$ and Mashonkina \& Vinogradova (2007), $S_{\mathrm{H}}=0.002$ for Al I and $S_{\mathrm{H}}=0.05$ for Na I according to Gehren et al. (2004), $S_{\mathrm{H}}=0.05$ for K I (Zhang et al. $2006 \mathrm{a}, \mathrm{b})$, and $S_{\mathrm{H}}=0.1$ for $\mathrm{Mg}$ I and Ca I/II as recommended by Mashonkina et al. (2007a).

\section{Line list and solar abundances for a differential analysis}

The spectral lines used in our study are listed in Table 3. Hyperfine structure (HFS) and/or isotope structure (IS) is taken into account when necessary with the data from Moore

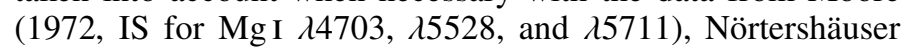
et al. (1998, IS for Ca II 18498), McWilliam et al. (1995, Sr II 14215), McWilliam (1998, Ba II 14554), and Lawler et al. (2001, $\mathrm{Eu}$ II 14129). For $\mathrm{Mg}, \mathrm{Ca}, \mathrm{Sr}$, and $\mathrm{Eu}$, we use the fractional isotope abundances corresponding to the solar system matter (Anders \& Grevesse 1989). For barium, the even-to-odd isotope abundance ratio was allowed to vary, and its value is determined in Sect. 7.2 for the two stars. 
Table 3. Atomic data and solar abundances for the investigated lines.

\begin{tabular}{|c|c|c|c|c|c|c|c|}
\hline Ion & $\lambda, \AA$ & $E_{\mathrm{exc}}$ & $\log g f$ & Reference & $\log C_{6}$ & Reference & $\log \varepsilon_{\odot}$ \\
\hline \multirow[t]{2}{*}{$\mathrm{NaI}$} & 5889.96 & 0.00 & 0.11 & 2 & -31.60 & 14 & 6.28 \\
\hline & 5895.93 & 0.00 & -0.19 & 2 & -31.60 & 14 & 6.28 \\
\hline \multirow[t]{6}{*}{ Mg I } & $4571.10^{1}$ & 0.00 & -5.62 & 2 & -31.96 & 14 & 7.58 \\
\hline & $4702.99^{1}$ & 4.33 & -0.44 & 2 & -29.71 & 14 & 7.49 \\
\hline & $5528.41^{1}$ & 4.33 & -0.50 & 2 & -30.20 & 14 & 7.53 \\
\hline & $5711.09^{1}$ & 4.33 & -1.72 & 2 & -29.89 & 14 & 7.55 \\
\hline & 5172.68 & 2.70 & -0.45 & 3 & -30.88 & 14 & 7.56 \\
\hline & 5183.60 & 2.70 & -0.24 & 3 & -30.88 & 14 & 7.57 \\
\hline $\mathrm{Al} \mathrm{I}$ & 3961.53 & 0.01 & -0.34 & 2 & -31.20 & 14 & 6.44 \\
\hline \multirow[t]{2}{*}{$\mathrm{K}_{\mathrm{I}}$} & 7664.92 & 0.00 & 0.13 & 4 & -31.00 & 5 & 5.12 \\
\hline & 7698.98 & 0.00 & -0.17 & 4 & -31.00 & 5 & 5.12 \\
\hline \multirow[t]{15}{*}{$\mathrm{Ca} \mathrm{I}$} & 4425.44 & 1.87 & -0.36 & 6 & -30.90 & $*$ & 6.41 \\
\hline & 4578.56 & 2.51 & -0.70 & 7 & -30.30 & 16 & 6.37 \\
\hline & 5261.71 & 2.51 & -0.58 & 7 & -30.86 & 16 & 6.44 \\
\hline & 5349.47 & 2.70 & -0.31 & 7 & -31.45 & 16 & 6.41 \\
\hline & 5588.76 & 2.51 & 0.36 & 7 & -31.39 & 16 & 6.28 \\
\hline & 5590.12 & 2.51 & -0.57 & 7 & -31.39 & 16 & 6.39 \\
\hline & 5857.45 & 2.92 & 0.24 & 7 & -30.61 & 16 & 6.36 \\
\hline & 6122.22 & 1.88 & -0.32 & 6 & -30.30 & 15 & 6.26 \\
\hline & 6162.17 & 1.89 & -0.09 & 6 & -30.30 & 15 & 6.28 \\
\hline & 6166.44 & 2.51 & -1.14 & 7 & -30.48 & 16 & 6.42 \\
\hline & 6439.07 & 2.51 & 0.39 & 7 & -31.58 & 16 & 6.27 \\
\hline & 6471.66 & 2.51 & -0.69 & 7 & -31.58 & 16 & 6.34 \\
\hline & 6493.78 & 2.51 & -0.11 & 7 & -31.58 & 16 & 6.31 \\
\hline & 6499.65 & 2.51 & -0.82 & 7 & -31.58 & 16 & 6.38 \\
\hline & 6449.81 & 2.51 & -0.50 & 7 & -31.45 & 16 & 6.34 \\
\hline \multirow{2}{*}{ Ca II } & 3933.66 & 0.00 & 0.10 & 8 & -31.72 & 17 & 6.35 \\
\hline & $8498.02^{1}$ & 1.69 & -1.42 & 8 & -31.51 & 17 & 6.35 \\
\hline \multirow[t]{11}{*}{ Fe II } & 4508.29 & 2.84 & -2.32 & 9 & -32.00 & 18 & 7.44 \\
\hline & 4582.84 & 2.83 & -3.14 & 9 & -32.03 & 18 & 7.43 \\
\hline & 4923.93 & 2.88 & -1.36 & 9 & -32.05 & 18 & 7.41 \\
\hline & 5018.44 & 2.88 & -1.23 & 9 & -32.05 & 18 & 7.45 \\
\hline & 5197.57 & 3.22 & -2.24 & 9 & -32.05 & 18 & 7.46 \\
\hline & 5234.62 & 3.21 & -2.14 & 9 & -32.05 & 18 & 7.41 \\
\hline & 5264.81 & 3.22 & -3.13 & 13 & -32.03 & 18 & 7.53 \\
\hline & 5325.56 & 3.21 & -3.26 & 12 & -32.05 & 18 & 7.56 \\
\hline & 5425.25 & 3.19 & -3.30 & 12 & -32.05 & 18 & 7.51 \\
\hline & 6247.56 & 3.87 & -2.43 & 13 & -32.00 & 18 & 7.57 \\
\hline & 6456.38 & 3.89 & -2.18 & 13 & -32.00 & 18 & 7.59 \\
\hline Sr II & $4215.53^{1}$ & 0.00 & -0.17 & 10 & -31.80 & $*$ & 2.92 \\
\hline \multirow[t]{2}{*}{ Ba II } & $4554.03^{1}$ & 0.00 & 0.16 & 10 & -31.65 & $*$ & 2.21 \\
\hline & 6496.90 & 0.60 & -0.38 & 10 & -31.28 & 17 & 2.21 \\
\hline Eu II & $4129.72^{1}$ & 0.00 & 0.22 & 11 & -32.08 & 19 & 0.56 \\
\hline
\end{tabular}

Excitation energy $E_{\mathrm{exc}}$ is given in $\mathrm{eV} ;{ }^{1}$ IS and HFS components are taken into account; reference: 2 - NIST database; 3 - Aldenius et al. (2007); 4 - Butler (2000); 5 - Zhang et al. (2006a); 6 - Smith \& O’Neil (1975); 7 - Smith \& Raggett (1981); 8 - Theodosiou (1989); 9 Landstreet (1998); 10 - Reader et al. (1980); 11 - Lawler et al. (2001); 12 - Moity (1983); 13 - Raassen \& Uylings (1998); 14 - Gehren et al. (2004); 15 - Anstee \& O'Mara (1995); 16 - Smith (1981); 17 - Barklem \& O’Mara (1998); 18 - Barklem \& Aspelund-Johansson (2005); 19 - Kurucz (1992); * - solar line profile fitting.

Based on non-LTE line formation, we determine the solar element abundance from each individual line. The exception is the Fe II lines, which are analyzed assuming LTE. The solar flux profiles from the Kurucz et al. (1984) Atlas are fitted using the fixed oscillator strengths and van der Waals damping constants collected in Table 3 . The profile of the strongest Ca line Ca II $\lambda 3933$ cannot be fitted although the best available atomic data are used. We assume that its core and inner wings are influenced by the chromospheric temperature rise and non-thermal depth-dependent chromospheric velocity field that is not part of the MAFAGS model. For this line, we adopt the mean Ca abundance derived from the $\mathrm{Ca}$ I lines.

We find that the mean solar abundances derived in this study agree within the error bars with the corresponding meteoritic abundances of Asplund et al. (2005). This gives confidence in the reliability of our results obtained in analysis of stellar spectra. Although analysis is made line-by-line differentially with respect to the Sun, it is not strictly differential. A line which is measurable in the selected metal-poor stars, is strong in the solar spectrum, and the derived solar element abundance depends not only on its $g f$-value but also on van der Waals damping parameter.

\section{Stellar parameters}

Our results both for stellar parameters and element abundances are based on line profile analysis. In order to compare with observations, computed synthetic profiles are convolved with a profile that combines instrumental broadening with a Gaussian profile and broadening by macroturbulence with a radial-tangential profile. For a given star, the macroturbulence value $V_{\text {mac }}$ was allowed to vary by $\pm 0.5 \mathrm{~km} \mathrm{~s}^{-1}(1 \sigma)$. Our strategy in this section is as follows.

1. Stellar effective temperature is determined from the hydrogen $\mathrm{H}_{\alpha}$ and $\mathrm{H}_{\beta}$ line wing fitting based on non-LTE line formation. As was shown by Fuhrmann et al. (1993), the Balmer line profiles are strongly temperature sensitive, while the variation with gravity and the metal abundance is rather small. $\mathrm{H}_{\alpha}$ in particular is very insensitive to $g$ and $[\mathrm{M} / \mathrm{H}]$. This is the basic justification for using the Balmer lines as a temperature indicator.

2. Next, the surface gravity is derived from the HIPPARCos parallax with a mass determined from the tracks of VandenBerg et al. (2000). The exception is $\mathrm{BD}-13^{\circ} 3442$ with no HIPPARCos parallax available.

3. The ionization balance between $\mathrm{Ca}$ I and $\mathrm{Ca}$ II is checked for the fixed temperature and current estimate of $\log g$. We denote the mean abundance from the subordinate lines of $\mathrm{Ca}$ I as the CaI abundance and from Ca II $\lambda 3933$ and Ca II $\lambda 8498$ as the CaII abundance. If (CaI-CaII) exceeds the abundance error, the gravity is revised. We note that the two Ca II lines behave oppositely with varying gravity. The resonance line has the van der Waals broadened wings even at $[\mathrm{Ca} / \mathrm{H}]<-2$, and the derived $\mathrm{Ca}$ abundance decreases/increases with increasing/decreasing gravity. The IR line is strengthened with decreasing $\log g$ due to decreasing the $\mathrm{H}^{-}$continuous absorption and due to amplified departures from LTE for Ca II. This gives an additional opportunity to constrain stellar gravity from a comparison of the element abundances from the two Ca II lines.

4. Simultaneously, the iron abundance is obtained from the Fe II lines assuming LTE, and the abundances of $\alpha$-process elements $\mathrm{Mg}$ and $\mathrm{Ca}$ are determined from non-LTE analysis of the $\mathrm{Mg} \mathrm{I}$ and $\mathrm{Ca} \mathrm{I}$ lines.

5. A microturbulence velocity $V_{\text {mic }}$ is derived from the strongest Fe II, $\mathrm{Ca}$ I, and $\mathrm{Mg}$ I lines sensitive at given stellar parameters to a variation in $V_{\text {mic }}$.

If any of the obtained parameters, either $T_{\text {eff }}$, or $\log g$, or $[\mathrm{Fe} / \mathrm{H}]$, or $\alpha$-enhancement, deviates from the one adopted originally, the model atmosphere is recalculated and the steps 1 through 5 are repeated. Thus, stellar parameters are determined 
iteratively. We describe below how it is made for the individual stars and order them by the level of the accuracy of stellar parameters $T_{\text {eff }}$ and $\log g$ available in the literature, from the higher accuracy to the lower one. The final stellar parameters and element abundances are presented in Table 4. The mean element abundance is given where more than one line is used in analysis, and the error bars quoted in Table 4 is computed as the standard deviation $\sigma=\sqrt{\Sigma\left(\bar{x}-x_{i}\right)^{2} /(n-1)}$.

\subsection{HD 84937}

This star with a bright magnitude of 8.28 is among the best studied halo stars. Its effective temperature was determined many times (for review, see Korn et al. 2003) both spectroscopically from Balmer line wing fitting and using the infrared flux method (IRFM) with surprisingly consistent results. According to the LTE analysis of Korn et al. (2003), $T_{\text {eff }}$ (Balmer) $=6346 \mathrm{~K}$, while Alonso et al. (1996) and Meléndez \& Ramírez (2004) give $T_{\text {eff }}($ IRFM) $=6330 \mathrm{~K}$ and $6345 \mathrm{~K}$, correspondingly. The HIPPARCos parallax, $\pi=12.44 \pm 1.06$ mas is known with an accuracy better than $10 \%$.

The Balmer line profiles are computed applying the two self-broadening recipes, $A G$ and $B P O$. In non-LTE, we obtain $T_{\text {eff }}(A G)=6380 \mathrm{~K}$ from $\mathrm{H}_{\alpha}$ and $6350 \mathrm{~K}$ from $\mathrm{H}_{\beta}$. Based on a $S / N$ ratio of the observed spectrum and a sensitivity of the Balmer lines to a variation of $T_{\text {eff }}$, we estimate the uncertainty of $T_{\text {eff }}$ arising from the profile fitting as $30 \mathrm{~K}$ for $\mathrm{H}_{\alpha}$ and $60 \mathrm{~K}$ for $\mathrm{H}_{\beta}$. The core within $\pm 1 \AA$ for $\mathrm{H}_{\alpha}$ and $\pm 0.7 \AA$ for $\mathrm{H}_{\beta}$ is not included in the fit. A mean temperature, $T_{\text {eff }}=6365 \mathrm{~K}$, is adopted as a final value. In the case of the self-broadening from Barklem et al. (2000), a mean temperature $T_{\text {eff }}(B P O)=6300 \mathrm{~K}$ is derived from two Balmer lines. The maximum error of $T_{\text {eff }}$ then is, probably, not larger than $70 \mathrm{~K}$.

A trigonometric gravity was carefully calculated by Korn et al. (2003), $\log g=4.00 \pm 0.12$. For a temperature $T_{\text {eff }}=$ $6365 \mathrm{~K}$ obtained in this study, $\log g$ increases by less than 0.01 . For the model with $T_{\text {eff }} / \log g=6365 / 4.00$, we find a good agreement between the non-LTE CaI abundance based on 14 lines of $\mathrm{CaI}$ and the non-LTE CaII abundance from two Ca II lines: $(\mathrm{CaI}-\mathrm{CaII})=0.01$. In contrary, an agreement between different lines is destroyed at the LTE assumption (see Table 4).

The error of the abundance difference (CaI-CaII) is mainly determined by the error of the CaII abundance, because the error of the CaI abundance is very small for the program stars. It constitutes $\sigma / \sqrt{n}$ where $n=15$ to 10 for different stars, and $\sigma$ does not exceed 0.06 dex (Table 4). For Ca II $\lambda 3933$ in different stars, the abundance error is mainly due to the uncertainties of the van der Waals damping constant and the profile fitting, while the uncertainty of microturbulence velocity dominates the abundance error for Ca II 18498 . The van der Waals damping constants predicted by the perturbation theory of Barklem \& O'Mara (1998) have the uncertainty $\Delta \log C_{6}=$ 0.05-0.18 dex according to Barklem \& Aspelund-Johansson (2005) and Barklem (2006). For Ca II $\lambda 3933$ in the program stars, this translates to the abundance error of up to $0.06 \mathrm{dex}$.

For Ca II $\lambda 3933$ in HD 84937, only the line wings are fitted because the observed Doppler core is very broad and most probably affected by the chromospheric temperature rise and nonthermal and depth-dependent chromospheric velocity fields similar to solar ones. The abundance error is estimated as $0.06 \mathrm{dex}$. For Ca II $\lambda 8498, \Delta V_{\text {mic }}=0.2 \mathrm{~km} \mathrm{~s}^{-1}$ leads to $\sigma=0.04 \mathrm{dex}$. Thus, the differences of LTE abundances (CaII (18498)-CaI)
Table 4. Stellar parameters and element abundances of the selected stars where $V_{\text {mic }}$ is given in $\mathrm{km} \mathrm{s}^{-1}$

\begin{tabular}{|c|c|c|c|c|}
\hline $\begin{array}{l}\mathrm{HD} / \mathrm{BD} \\
\end{array}$ & 122563 & 84937 & $+3^{\circ} 740$ & $-13^{\circ} 3442$ \\
\hline$T_{\mathrm{eff}}, \mathrm{K}$ & 4600 & 6365 & 6340 & 6390 \\
\hline $\log g$ & $1.50 \pm 0.2$ & $4.00 \pm 0.12$ & $3.90 \pm 0.15$ & $3.88 \pm 0.15$ \\
\hline$[\mathrm{Fe} / \mathrm{H}]$ & $-2.53 \pm 0.02$ & $-2.15 \pm 0.04$ & $-2.65 \pm 0.01$ & $-2.66 \pm 0.01$ \\
\hline \multirow[t]{2}{*}{$V_{\text {mic }}$} & 1.9 & 1.6 & 1.4 & 1.4 \\
\hline & NLTE & & & \\
\hline$[\mathrm{Mg} / \mathrm{H}]$ & $-2.22 \pm 0.06$ & $-1.76 \pm 0.04$ & $-2.35 \pm 0.03$ & $-2.26 \pm 0.05$ \\
\hline$[\mathrm{Na} / \mathrm{H}]$ & $-2.93 \pm 0.01$ & $-2.48 \pm 0.02$ & $-3.22 \pm 0.03$ & $-2.82 \pm 0.01$ \\
\hline$[\mathrm{Al} / \mathrm{H}]$ & -3.09 & -2.21 & & -2.61 \\
\hline$[\mathrm{CaI} / \mathrm{H}]$ & $-2.32 \pm 0.06$ & $-1.72 \pm 0.04$ & $-2.19 \pm 0.05$ & $-2.12 \pm 0.06$ \\
\hline \multicolumn{5}{|l|}{$[\mathrm{CaII} / \mathrm{H}]$} \\
\hline$(\lambda 3933)$ & -2.42 & -1.70 & -2.16 & -2.15 \\
\hline$(\lambda 8498)$ & -2.36 & -1.76 & -2.14 & -2.06 \\
\hline$[\mathrm{K} / \mathrm{H}]$ & $-2.60 \pm 0.04$ & $-1.87 \pm 0.05$ & $-2.37 \pm 0.04$ & -2.28 \\
\hline$[\mathrm{Ba} / \mathrm{H}]$ & -3.54 & -2.15 & 2.85 & -2.89 \\
\hline$[\mathrm{Sr} / \mathrm{H}]$ & -2.93 & -2.18 & -2.62 & -2.35 \\
\hline \multirow[t]{2}{*}[\mathrm{Eu}/\mathrm{H}]{} & -3.04 & -1.45 & & \\
\hline & LTE & & & \\
\hline$[\mathrm{Mg} / \mathrm{H}]$ & $-2.39 \pm 0.10$ & $-1.88 \pm 0.03$ & $-2.40 \pm 0.05$ & $-2.29 \pm 0.03$ \\
\hline$[\mathrm{Na} / \mathrm{H}]$ & $-2.51 \pm 0.04$ & $-2.02 \pm 0.03$ & $-3.03 \pm 0.02$ & $-2.58 \pm 0.08$ \\
\hline$[\mathrm{Al} / \mathrm{H}]$ & -3.13 & -3.04 & & -3.47 \\
\hline$[\mathrm{CaI} / \mathrm{H}]$ & $-2.52 \pm 0.10$ & $-1.76 \pm 0.04$ & $-2.32 \pm 0.07$ & $-2.27 \pm 0.06$ \\
\hline \multicolumn{5}{|l|}{$[\mathrm{CaII} / \mathrm{H}]$} \\
\hline$(\lambda 3933)$ & -2.42 & -1.70 & -2.16 & -2.15 \\
\hline$(\lambda 8498)$ & -2.29 & -1.56 & -1.92 & -1.81 \\
\hline$[\mathrm{K} / \mathrm{H}]$ & $-2.25 \pm 0.02$ & $-1.65 \pm 0.03$ & $-2.17 \pm 0.04$ & -2.08 \\
\hline$[\mathrm{Ba} / \mathrm{H}]$ & -3.57 & -2.30 & -3.32 & -3.39 \\
\hline$[\mathrm{Sr} / \mathrm{H}]$ & -2.85 & -2.18 & -2.89 & -2.57 \\
\hline$[\mathrm{Eu} / \mathrm{H}]$ & -3.16 & -1.61 & & \\
\hline
\end{tabular}

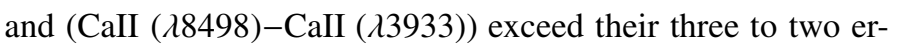
rors.

The iron abundance $[\mathrm{Fe} / \mathrm{H}]=-2.15$ and microturbulence velocity $V_{\text {mic }}=1.6 \mathrm{~km} \mathrm{~s}^{-1}$ are determined from $10 \mathrm{Fe}$ II lines requiring that the derived abundances do not depend on line strength. The $\alpha$-enhancement of the model is defined by the nonLTE $\mathrm{Mg}$ abundance, $[\mathrm{Mg} / \mathrm{Fe}]=0.39$.

\section{2. $H D 122563$}

HD 122563 is one of the best observed halo stars. The SIMBAD Astronomical Database finds more than 300 references to studies of this star since 1980. However, stellar parameters derived by different authors show a large spread of data. Most papers rely on an infrared flux method temperature $T_{\text {eff }}($ IRFM) $=4572 \mathrm{~K}$, as determined by Alonso et al. (1999). Fulbright (2000) gets the lower value, $T_{\text {eff }}=4425 \mathrm{~K}$, requiring the same iron abundance from the Fe Ilines with high and lower excitation potential. A mean temperature $T_{\text {eff }}=4615 \mathrm{~K}$ is deduced by Barbuy et al. (2003) from using the $b-y, V-R, V-K$, and $J-K$ calibrations of Alonso et al. (1999). Surface gravity derived in different studies, either from the HIPPARCOS parallax or from the ionization balance between Fe I and Fe II, ranges between $\log g=1.5$ (Barbuy et al. 2003) and $\log g=0.6$ (Fulbright 2000). Significant discrepancies are seen for the microturbulence velocity: $V_{\text {mic }}$ varies between $2 \mathrm{~km} \mathrm{~s}^{-1}$ (Barbuy et al. 2003) and $V_{\text {mic }}=2.9 \mathrm{~km} \mathrm{~s}^{-1}$ (Simmerer et al. 2004).

In spectroscopic determination of $T_{\mathrm{eff}}$, we use the observed spectrum taken from the ESO UVESPOP survey (Bagnulo et al. 2005). The wings of $\mathrm{H}_{\alpha}$ and $\mathrm{H}_{\beta}$ in $\mathrm{HD} 122563$ are rather weak as compared to the turnoff stars. However, they are still sensitive to $T_{\text {eff }}$. This is illustrated in Fig. 3. Non-LTE leads to a consistency 

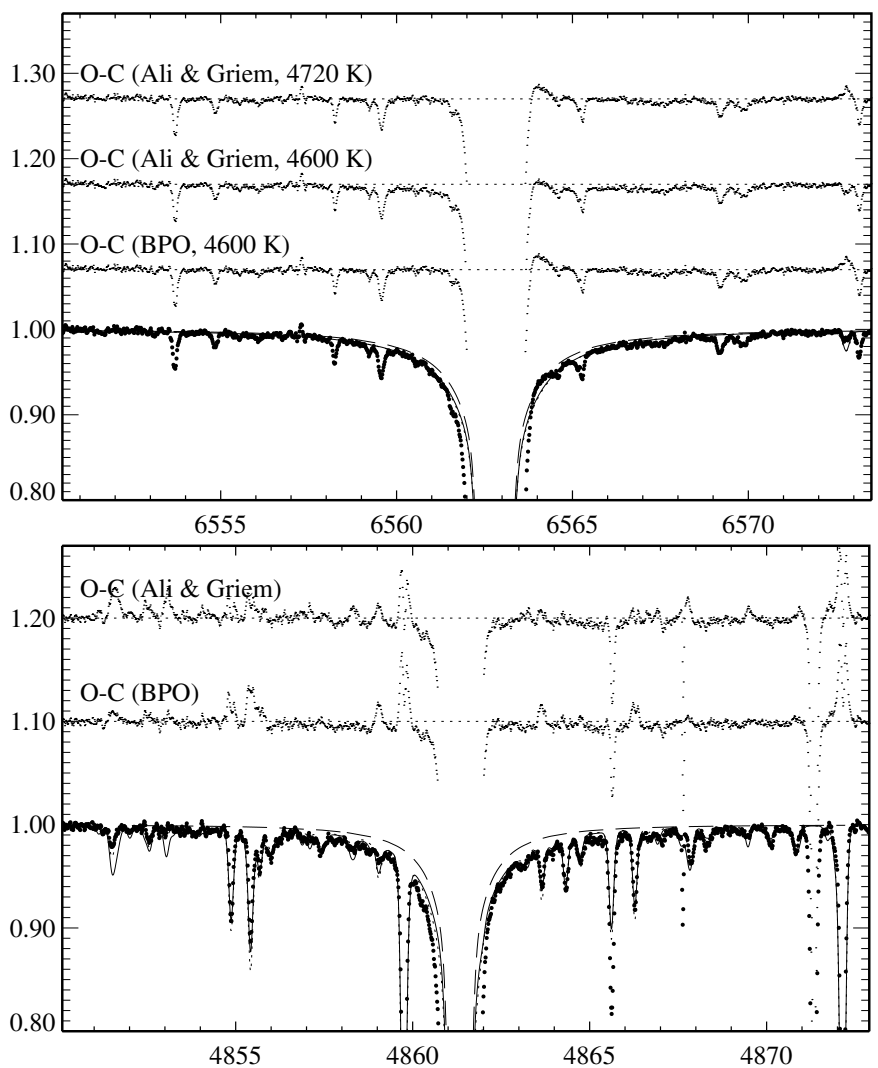

Fig. 3. Theoretical non-LTE flux profiles computed using the $A G$ (continuous line for $T_{\text {eff }}=4720 \mathrm{~K}$, dashed line for $T_{\text {eff }}=4600 \mathrm{~K}$ ) and $B P O$ (dotted line, $T_{\text {eff }}=4600 \mathrm{~K}$ ) self-broadening recipes compared to the observed UVES profiles (bold dots) of $\mathrm{H}_{\alpha}$ (top panel) and $\mathrm{H}_{\beta}$ (bottom panel) in HD 122563. The (O-C) values are shown in the upper part of each panel.

of the temperatures derived from the two Balmer lines. For the two broadening recipes, we find $T_{\text {eff }}(A G)=4720 \mathrm{~K}$ and $T_{\text {eff }}(B P O)=4600 \mathrm{~K}$. The uncertainty of $T_{\text {eff }}$ arising from the profile fitting is estimated as $60 \mathrm{~K}$ for $\mathrm{H}_{\alpha}$ and $50 \mathrm{~K}$ for $\mathrm{H}_{\beta}$ based on a $S / N$ ratio of the observed spectrum and a sensitivity of the Balmer lines to a variation of $T_{\text {eff }}$. The line core within $\pm 1.5 \AA$ for $\mathrm{H}_{\alpha}$ and within $\pm 1.2 \AA$ for $\mathrm{H}_{\beta}$ is not included in the fit. Figure 3 shows the best fits and the differences between observed and calculated spectra, $(\mathrm{O}-\mathrm{C})$. At the LTE assumption, $\mathrm{H}_{\alpha}$ gives a $60 \mathrm{~K}$ lower temperature.

As a final value we adopt $T_{\text {eff }}=4600 \mathrm{~K}$ that is supported both spectroscopically and photometrically. The uncertainty of $120 \mathrm{~K}$ is estimated as the difference between $T_{\text {eff }}$ from the two broadening recipes, because a treatment of the self-broadening of the hydrogen lines turns out to be the most significant source of $T_{\text {eff }}$ errors. The effect of $T_{\text {eff }}$ variation by $120 \mathrm{~K}$ on metal abundances is shown in Table 5 . We note that a significant discrepancy of 0.33 dex appears between $\operatorname{Mg}$ I $\lambda 4571$ and the remaining five $\mathrm{Mg}$ I lines when $T_{\text {eff }}$ increases by $120 \mathrm{~K}$. This is due to a larger increase of departures from LTE for the line arising from the ground state compared to that for the subordinate lines.

A trigonometric gravity based on the HIPPARCos parallax, $\pi=3.76 \pm 0.72$ mas, was carefully calculated by Barbuy et al. (2003) using $T_{\text {eff }}=4600 \mathrm{~K}, \log g=1.53 \pm 0.2$. Based on nonLTE line formation, we check the ionization balance between Ca I and Ca II for three gravitis, $\log g=1.50,1.30$, and 1.70, in order to find a right value. In each case, we determine the
Table 5. Effect on element abundances (in dex) and the Ba odd isotope fraction $f_{\text {odd }}$ (in \%) of HD 122563 caused by uncertainties of its stellar parameters.

\begin{tabular}{lcccc}
\hline \hline Chemical & $T_{\text {eff }}(\mathrm{K})$ & \multicolumn{2}{c}{$\log g$} & $V_{\text {mic }}\left(\mathrm{km} \mathrm{s}^{-1}\right)$ \\
\cline { 3 - 4 } species & +120 & -0.2 & +0.2 & +0.1 \\
\hline Fe II & $<+0.01$ & -0.07 & +0.08 & $<0.01$ \\
Ca I & +0.11 & +0.03 & -0.03 & $<0.01$ \\
Ca II & & & & \\
$(\lambda 3933)$ & +0.11 & +0.02 & -0.01 & $<0.01$ \\
$(\lambda 8498)$ & +0.12 & +0.00 & +0.09 & $<0.01$ \\
$\mathrm{Mg}_{\text {I }}$ & +0.11 & +0.03 & -0.03 & -0.02 \\
$\mathrm{Ba}_{\text {II }}$ & +0.07 & -0.08 & +0.08 & $<0.01$ \\
$f_{\text {odd }}$ & +12 & +2 & -3 & -8 \\
\hline
\end{tabular}

iron abundance from 9 lines, the $\mathrm{Mg}$ abundance from 6 lines, the $\mathrm{CaI}$ abundance from 15 lines, and the microturbulence velocity from the strongest lines of Fe II (multiplet 42), Mg I (5 lines), and Ca I (3 lines). The Ca II resonance line is most probably affected by the chromospheric temperature rise, and the CaII abundance of HD 122563 is obtained only from Ca II $\lambda 8498$ which is believed to be of photospheric origin. Strong departures from LTE are found for Ca II $\lambda 8498$, such that, with the LTE assumption, its profile cannot be fitted by a variation in the $\mathrm{Ca}$ abundance. The LTE abundance is determined from line wing fits as shown in Fig. 4. We note that the theoretical LTE profile in Fig. 4 (top panel) has been computed for an 0.07 dex larger $\mathrm{Ca}$ abundance, compared to that for the non-LTE profile, and this only helps to reproduce the wings but not the core of Ca II 18498 . If to use the equivalent width of $\lambda 8498$, LTE leads to a 0.18 dex overestimated $\mathrm{Ca}$ abundance. In non-LTE, the core of $\lambda 8498$ in HD 122563 is better fitted though not perfectly. The line is saturated, and its half-width is only weakly sensitive to a variation of the $\mathrm{Ca}$ abundance and $V_{\text {mic }}$. We rely therefore on fitting the observed profile beyond $\Delta \lambda=0.4 \AA$. The uncertainty of the profile fitting translates to the abundance error of $0.06 \mathrm{dex}$. The maximal error of the van der Waals damping constant $\Delta \log C_{6}=0.2$ leads to $\Delta \log \varepsilon=0.06 \mathrm{dex}$.

The effect of gravity variation on the abundance of $\mathrm{Ca}$ from two ionization stages is shown in Table 5. We find that the CaI and CaII abundances are consistent within the error bars for each of three gravities. The abundance difference is minimal for $\log g=1.50,(\mathrm{CaI}-\mathrm{CaII})=+0.04 \mathrm{dex}$ and equals $+0.07 \mathrm{dex}$ and -0.08 dex for $\log g=1.30$ and 1.7 , correspondingly. Thus, a trigonometric gravity is supported spectroscopically, and we adopt $\log g=1.50 \pm 0.2$ as a final value.

\section{3. $B D+3^{\circ} 740$}

For $\mathrm{BD}+3^{\circ} 740$, we note a surprisingly large discrepancy between the infrared flux method temperatures derived by Alonso et al. (1996), $T_{\text {eff }}($ IRFM) $=6110 \mathrm{~K}$, and Meléndez \& Ramírez $(2004), T_{\text {eff }}($ IRFM) $=6440 \mathrm{~K}$. An effective temperature determined in different studies from different spectroscopic and photometric methods (for review, see Ivans et al. 2003) varies between $T_{\text {eff }}=6000 \mathrm{~K}$ (Ivans et al. 2003) and $T_{\text {eff }}=6355 \mathrm{~K}$ (Carretta et al. 2002).

Based on non-LTE line formation for H I with hydrogen collisions taken into account applying $S_{\mathrm{H}}=2$, we obtain from both Balmer lines $T_{\text {eff }}(A G)=6340 \mathrm{~K}$ and $T_{\text {eff }}(B P O)=6260 \mathrm{~K}$. The uncertainty of $T_{\text {eff }}$ arising from the profile fitting is estimated as $70 \mathrm{~K}$ for $\mathrm{H}_{\alpha}$ and $80 \mathrm{~K}$ for $\mathrm{H}_{\beta}$. The core within $\pm 1.2 \AA$ for $\mathrm{H}_{\alpha}$ and within $\pm 0.7 \AA$ for $\mathrm{H}_{\beta}$ is not included in the fit. Figure 5 

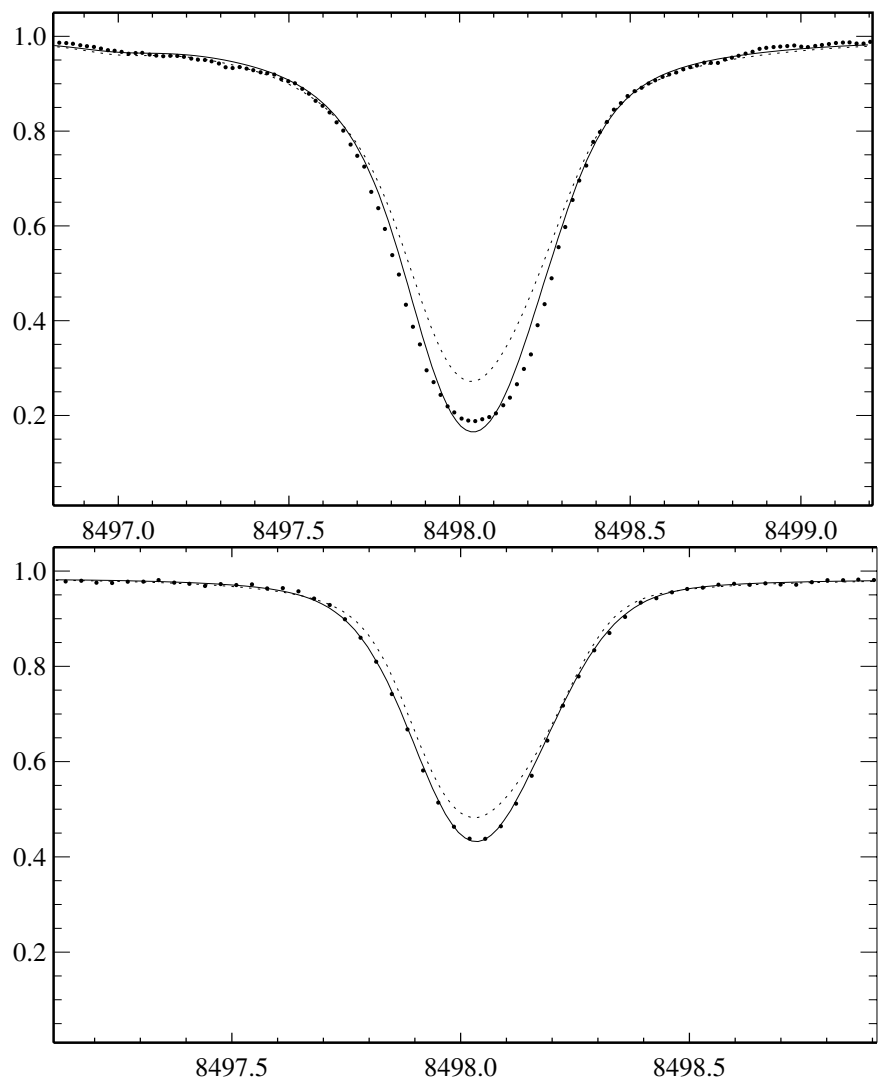

Fig. 4. The best non-LTE $\left(S_{\mathrm{H}}=0.1\right.$, continuous line) and LTE (dotted line) fits of the observed profiles (bold dots) of Ca II $\lambda 8498$ in HD 122563 (top panel) and BD+3 740 (bottom panel). The obtained non-LTE and LTE abundances of Ca are presented in Table 4. Note, that it is impossible to achieve well fitting in LTE.

shows the best fits and the sensitivity of the Balmer line profiles to a $100 \mathrm{~K}$ variation in $T_{\text {eff }}$. For the LTE calculations, nearly the same quality fit of $\mathrm{H}_{\alpha}$ is achieved at a $60 \mathrm{~K}$ lower temperature, however, provided that the core within $\pm 1.7 \AA$ is not included in the fit. In the inner wings $(\Delta \lambda=1.2 \AA-1.7 \AA$ ), the LTE profile is too broad for $T_{\text {eff }}$ obtained from the far wings (see Fig. 5). When non-LTE calculations are performed with reduced hydrogenic collisions $\left(S_{\mathrm{H}}=0.1\right)$, a $100 \mathrm{~K}$ larger effective temperature is determined from the $\mathrm{H}_{\alpha}$ wings, beyond $2 \AA$ from the line center, while the inner wings are obtained to be too narrow. Thus, the uncertainty of $T_{\text {eff }}\left(\mathrm{H}_{\alpha}\right)$ due to various treatment of hydrogen line formation is estimated as $(+100 \mathrm{~K},-60 \mathrm{~K})$. Having in mind that non-LTE effects are negligible for $\mathrm{H}_{\beta}$, a consistency of the temperatures derived from two Balmer lines favors the non-LTE approach with hydrogen collisions taken into account with $S_{\mathrm{H}}$ of 1 to 2 .

As a final value, $T_{\text {eff }}=6340 \mathrm{~K}$, is adopted with the uncertainty of $100 \mathrm{~K}$. The effect of $T_{\text {eff }}$ variation on metal abundances is shown in Table 6.

According to Fuhrmann (1998), a trigonometric gravity corresponding to the HIPPARCos parallax, $\pi=7.80 \pm 2.09$ mas, is inferred as $\log g=4.18$, and it can lie between $\log g=3.92$ and $\log g=4.37$ due to the relatively large error of the parallax. Asplund et al. (2006) use the mean value of the absolute visual magnitude derived from the HIPPARCos parallax and from Strömgren photometry and obtain $\log g=4.04$. In both studies, $T_{\text {eff }}=6260 \mathrm{~K}$ is used. We have checked the ionization balance between Ca I and Ca II for $\log g=4.06$ and 3.90, applying
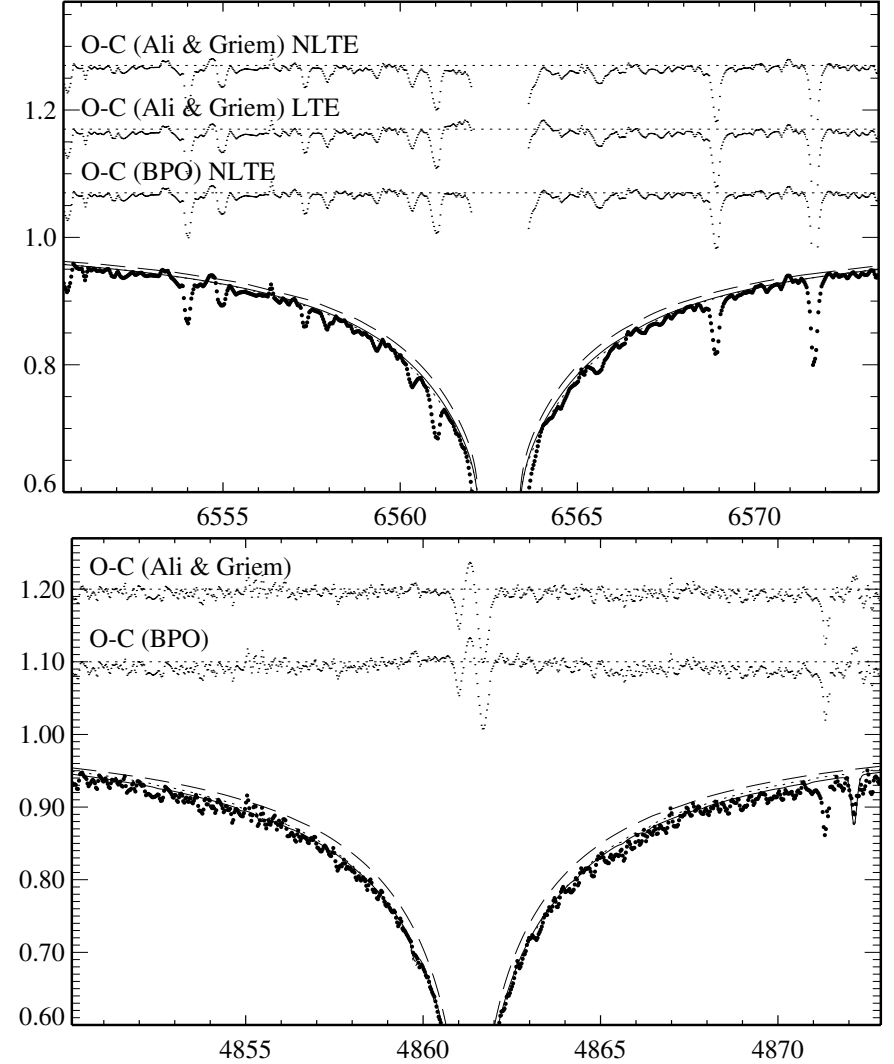

Fig. 5. The best non-LTE fits of $\mathrm{H}_{\alpha}$ (top panel) and $\mathrm{H}_{\beta}$ (bottom panel) in the observed FOCES spectrum (bold dots) of $\mathrm{BD}+3^{\circ} 740$ achieved using the $A G$ (continuous line, $\left.T_{\text {eff }}=6340 \mathrm{~K}\right)$ and $B P O\left(\right.$ dotted line, $T_{\mathrm{eff}}=$ $6260 \mathrm{~K})$ self-broadening recipes. For comparison, we show $(\mathrm{O}-\mathrm{C})$ also for the best LTE $A G$ fit of $\mathrm{H}_{\alpha}$ computed for $T_{\text {eff }}=6280 \mathrm{~K}$. Note that the LTE inner wings $(\Delta \lambda=1.2-1.6 \AA)$ are broader compared to the nonLTE ones although the lower effective temperature is used in the LTE calculations. In order to illustrate the sensitivity of the line profiles to $T_{\text {eff }}$, the non-LTE $A G$ profiles are shown also for $T_{\text {eff }}=6240 \mathrm{~K}$ (dashed curves).

Table 6. Effect on element abundances (in dex) of $\mathrm{BD}+3^{\circ} 740$ and $\mathrm{BD}-13^{\circ} 3442$ caused by uncertainties of its stellar parameters

\begin{tabular}{lcccc}
\hline \hline Chemical & $T_{\text {eff }}(\mathrm{K})$ & \multicolumn{2}{c}{$\log g$} & $V_{\text {mic }}\left(\mathrm{km} \mathrm{s}^{-1}\right)$ \\
\cline { 3 - 4 } species & -100 & +0.16 & -0.28 & +0.2 \\
\hline Fe II & -0.02 & +0.05 & -0.09 & $<0.01$ \\
Ca I & -0.06 & +0.02 & +0.01 & $<0.01$ \\
Ca II & & & & \\
$(\lambda 3933)$ & -0.10 & -0.03 & +0.04 & $<0.01$ \\
$(\lambda 8498)$ & -0.08 & +0.06 & -0.05 & -0.06 \\
\hline
\end{tabular}

$T_{\text {eff }}=6340 \mathrm{~K}$. Table 6 presents the effect of gravity varying on the obtained abundance of $\mathrm{Ca}$. The $\mathrm{CaI}$ abundance is determined from 10 lines with the measurement error $\sigma=0.05$ dex. For Ca II $18498, \Delta V_{\text {mic }}=0.2 \mathrm{~km} \mathrm{~s}^{-1}$ leads to the abundance error of 0.06 dex, while the uncertainty of the profile fitting to $0.03 \mathrm{dex}$. For the Ca II resonance line, the total abundance error is estimated as 0.06 dex.

For $\log g=3.90$, we find a consistency within 0.04 dex of the $\mathrm{CaI}$ and $\mathrm{CaII}$ abundances. For the higher gravity, an agreement between the different lines of $\mathrm{Ca}$ is destroyed: CaII ( 18498) $-\mathrm{CaI}=0.09$ dex and CaII $($ 18498) $-\mathrm{CaII}(\lambda 3933)=$ 0.11 dex. As a final value, we adopt $\log g=3.90 \pm 0.15$ which is the lower limit of a trigonometric gravity and which is best supported spectroscopically. 


\section{4. $B D-13^{\circ} 3442$}

This is the faintest star of our small sample with no HIPPARCos parallax measured. In the observed UVES spectrum of this star, only $\mathrm{H} \alpha$ can be used to derive a temperature. The obtained spectroscopic temperature based either on the Ali \& Griem (1966) theory, $T_{\text {eff }}(A G)=6390 \mathrm{~K}$, or on the $B P O$ recipe, $T_{\text {eff }}(B P O)=$ $6310 \mathrm{~K}$, is lower compared to an infrared flux method temperature, $T_{\text {eff }}($ IRFM $)=6484 \mathrm{~K}$, as determined by Meléndez \& Ramírez (2004). As a final value, $T_{\text {eff }}=6390 \mathrm{~K}$ is adopted with the uncertainty similar to that for $\mathrm{BD}+3^{\circ} 740,100 \mathrm{~K}$.

Using the absolute visual magnitude from Strömgren photometry, Asplund et al. (2006) obtain $\log g=3.86$ for $\mathrm{BD}-13^{\circ} 3442$. A 0.02 dex larger surface gravity is obtained for a slightly higher temperature, $T_{\text {eff }}=6390 \mathrm{~K}$, determined in this study. Assuming that the gravity error is similar to that found for $\mathrm{BD}+3^{\circ} 740$, we check the ionization balance between $\mathrm{Ca}$ I and Ca II for $\log g=3.88$ and 3.60. For each of two gravities, the $\mathrm{CaI}$ and $\mathrm{CaII}$ abundances turn out to be consistent within the error bars. The effect of a gravity variation on the obtained abundances of $\mathrm{Ca}$ is shown in Table 6. We adopt $\log g=3.88$ as a final value. It is worth noting that the calculated atmospheric parameters of $\mathrm{BD}-13^{\circ} 3442$ and $\mathrm{BD}+3^{\circ} 740$ are very similar.

It is interesting to inspect the position of the selected stars on evolutionary tracks using the obtained stellar parameters. The grid of stellar models of VandenBerg et al. (2000) is used. According to interpolation along lines of constant radii in a cube of masses, metal abundances and $[\alpha / \mathrm{Fe}]$ ratios for available tracks we derive the HRD position of HD 84937 with a mass of $0.784 M_{\odot}$ and an age of $14.0 \mathrm{Gyr}$ (linear interpolation) or an age of 13.7 Gyr (logarithmic interpolation). The age determined for the known trigonometric parallax is slightly higher, i.e. $15.3 \mathrm{Gyr}$. The other two turnoff stars, $\mathrm{BD}+3^{\circ} 740$ and $\mathrm{BD}-13^{\circ} 3442$, are outside the set of evolutionary tracks with their low metal abundances. Their masses and ages can only be estimated by extrapolation (the lowest metal abundance of the grid is at $[\mathrm{Fe} / \mathrm{H}]=$ -2.3). Their masses are $0.766 M_{\odot}\left(\mathrm{BD}+3^{\circ} 740\right)$ and $0.790 M_{\odot}$ $\left(\mathrm{BD}-13^{\circ} 3442\right)$, whereas their ages are $14.9 \mathrm{Gyr}\left(\mathrm{BD}+3^{\circ} 740\right)$ and 13.0 Gyr (BD-13 3442$)$. The estimated ages are acceptable in view of the WMAP results that center around an age of the universe of 13.7 Gyr (Spergel et al. 2003). HD 122563 lies on the giant branch. In such case, extrapolation leads to too uncertain results for stellar ages.

\section{Abundance analysis}

In order to derive aluminium and potassium abundances, we use the UVES archive spectra because the AlI $\lambda 3961$ and K I $\lambda \lambda 7665,7699$ lines are not covered by the available Subaru spectra. For $\mathrm{BD}+3^{\circ} 740$, we cannot calculate $\mathrm{Al}$ abundance from the line at $\lambda 3961 \AA$ because the observed spectrum in this region is missing. The $\mathrm{KI} \lambda 7699$ line in $\mathrm{BD}-13^{\circ} 3442$ is blended with telluric lines and therefore is excluded from analysis. In $\mathrm{BD}+3^{\circ} 740$ and $\mathrm{BD}-13^{\circ} 3442$, the Eu II $\lambda 4129$ and $\mathrm{Ba}$ II $\lambda 6496$ lines cannot be extracted from noise. The $\mathrm{Ba}$ abundance of these stars is obtained from a single line, Ba II $\lambda 4554$, assuming a pure $r$-process even-to-odd $\mathrm{Ba}$ isotope ratio of 54:46 (Arlandini et al. 1999). It is worth noting that the Ba II line in these stars is weak $\left(W_{\lambda} \sim 10 \mathrm{m \AA}\right)$ and is insensitive to a variation of Ba isotopic abundances. The derived non-LTE and LTE abundances are presented in Table 4.

For the selected elements, non-LTE abundances of the two stars were determined in our earlier studies based on the FOCES observed spectra (Gehren et al. 2006, $\mathrm{Na}$ and $\mathrm{Mg}$ in

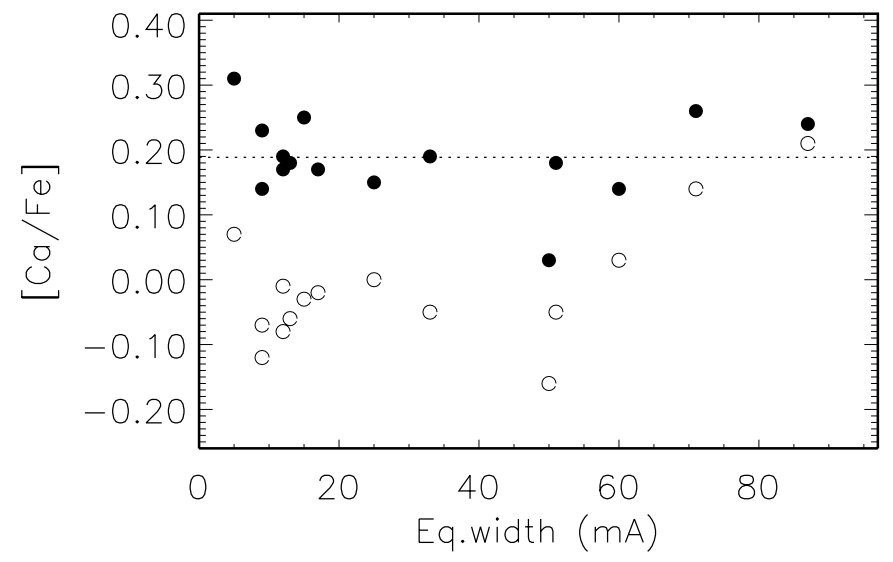

Fig. 6. Trends of non-LTE (filled circles) and LTE (open circles) abundance with line strength determined from the Ca I lines in HD 122563. Note the steep trend of the LTE $[\mathrm{Ca} / \mathrm{Fe}]$ values with line strength above $W_{\lambda}=60 \mathrm{~m} \AA$. The mean non-LTE value is shown by dotted line.

HD 84937 and BD+3740; Mashonkina et al. 2007a, Mg and Ca in HD 84937). Shimanskaya \& Mashonkina (2001) calculated the non-LTE abundances of Mg for HD 84937 and BD $+3^{\circ} 740$ based on the equivalent widths from the literature. In this paper, we obtain very similar results.

We find that HD 122563 reveals significantly lower element/Fe abundance ratios compared to the remaining stars. For the most investigated elements, there is underabundance by 1.2 dex for $\mathrm{Eu}$, by more than 0.75 dex for $\mathrm{Ba}, 0.5$ dex for $\mathrm{Al}$, 0.3 dex for $\mathrm{K}$ and $\mathrm{Sr}$, and 0.2 dex for $\mathrm{Ca}$. This reflects most probably a chemical inhomogeneity of the interstellar medium in the early Galaxy. An environment, where HD 122563 was formed, was enriched with the neutron-capture elements and the elements with odd nuclear charge $\mathrm{Al}$ and $\mathrm{K}$ to the less extent compared to the interstellar gas out of which the remaining stars were formed.

\subsection{Departures from LTE}

Significant non-LTE effects are found for almost all lines in our program stars. Non-LTE abundance corrections $\Delta_{\mathrm{NLTE}}=$ $\log \varepsilon_{\text {non-LTE }}-\log \varepsilon_{\text {LTE }}$ are negative for alkali atoms $\mathrm{Na}$ I and $\mathrm{K}$ I. They grow in absolute value with the strength of the observed line, i.e. with decreasing effective temperature. The maximal values are reached for HD 122563: $\Delta_{\mathrm{NLTE}}(\mathrm{NaI})=-0.44$ dex and $\Delta_{\text {NLTE }}(\mathrm{KI})=-0.35$ dex.

For the photoionization dominated minority species $\mathrm{Mg} \mathrm{I}$, $\mathrm{Al} \mathrm{I}$, and $\mathrm{Ca} \mathrm{I}, \Delta_{\mathrm{NLTE}}$ is positive. We note a strong temperature dependence of departures from LTE for Al I. Non-LTE abundance correction is equal 0.86 dex for the hottest star BD-13 3442 , while consists of only 0.04 dex for the coolest star HD 122563. For the $\mathrm{Mg}$ I and CaIlines, $\Delta_{\mathrm{NLTE}}$ does not exceed 0.2 dex. However, an advantage of the non-LTE approach is clearly seen in Fig. 6 where we show the $\mathrm{Ca}$ abundances calculated from the neutral Ca lines in HD 122563. Non-LTE removes the steep trend of the $[\mathrm{Ca} / \mathrm{Fe}]$ values with line strength obtained under the LTE assumption above $W_{\lambda}=60 \mathrm{~m} \AA$.

As was found theoretically in our earlier studies, non-LTE may lead either to strengthening or to weakening the Ba II (Mashonkina et al. 1999) and Sr II (Mashonkina \& Gehren 2001) lines depending on stellar parameters and element abundance. This is nicely illustrated with our small sample of stars. A slightly negative non-LTE abundance correction is obtained for 
Sr II $\lambda 4215$ in HD 122563 and a positive one for two the most metal-poor stars, while $\Delta_{\mathrm{NLTE}}=0$ for HD 84937 . For the Ba II lines in our program stars, $\Delta_{\mathrm{NLTE}}$ is positive and reaches $0.50 \mathrm{dex}$ for BD $-13^{\circ} 3442$. Non-LTE effects lead to $\Delta_{\mathrm{NLTE}}=+0.16 \mathrm{dex}$ for $\mathrm{Eu}$ II $\lambda 4129$.

\subsection{Comparisons with non-LTE studies}

For the elements under unvestigation, systematic analyses based on non-LTE line formation are few in number. We find that the non-LTE abundances obtained in this paper agree within the error bars with the corresponding abundances in the literature for common stars. For the three stars, HD $84937, \mathrm{BD}+3^{\circ} 740$, and BD- $13^{\circ} 3442$, the absolute abundance difference (this study Idiart \& Thevenin 2000) does not exceed 0.08 dex for $\mathrm{Mg}$ and 0.12 dex for $\mathrm{Ca}$. The non-LTE abundances of $\mathrm{Mg}$ and $\mathrm{Ca}$ given by Gratton et al. (2003) for HD 84937 are consistent within 0.06 dex with ours.

The non-LTE abundance of Na determined by Mishenina et al. (2003) for HD 84937 agrees within the error bars with our data. For HD 122563, we derive a 0.14 dex larger Na abundance compared to that given by Andrievsky et al. (2007). This is most probably due to the lower surface gravity $(\log g=1.1)$ used in their study and, therefore, the stronger non-LTE effects for the $\mathrm{Na}$ I lines.

The non-LTE abundance of potassium obtained by Takeda et al. (2002) agrees within 0.03 dex with our value for HD 122563.

The non-LTE abundances of $\mathrm{Al}$ and heavy elements $\mathrm{Sr}, \mathrm{Ba}$, and $\mathrm{Eu}$ are determined for the first time for the program stars.

\subsection{Galactic abundance trends: non-LTE vs. LTE}

In the view of significant departures from LTE found for the program stars, it is important to investigate what might change from the abundance trends determined from the larger samples of stars analyzed in LTE in the literature.

The $\alpha$-process elements, $\mathrm{Mg}$ and $\mathrm{Ca}$. There are well established observational evidences for that, in old metal-poor $([\mathrm{Fe} / \mathrm{H}]<-1)$ stars of the Galaxy, $\mathrm{Mg}$ and $\mathrm{Ca}$ are overabundant relative to iron (see e.g. recent determinations of Fulbright 2000; Cayrel et al. 2004; Cohen et al. 2004; Barklem et al. 2005, they all computed under LTE). The different samples of stars reveal close together average abundance ratios for $\mathrm{Mg} / \mathrm{Fe}$ and $\mathrm{Ca} / \mathrm{Fe}$ and the small scatter of data at the level of the measurement errors. E.g. Cohen et al. (2004) obtain the mean abundances $[\mathrm{Mg} / \mathrm{Fe}]=0.39 \pm 0.13$ and $[\mathrm{Ca} / \mathrm{Fe}]=0.31 \pm 0.12$ for the sample of the turnoff stars with $-3.5 \leq[\mathrm{Fe} / \mathrm{H}] \leq-2$, and Cayrel et al. (2004) find $[\mathrm{Mg} / \mathrm{Fe}]=0.27 \pm 0.13$ and $[\mathrm{Ca} / \mathrm{Fe}]=0.33 \pm 0.11$ for the sample of the cool giants with $-4 \leq[\mathrm{Fe} / \mathrm{H}] \leq-2.4$. In the range of stellar parameters the studies are concerned with, non-LTE leads to larger abundances of $\mathrm{Mg}$ and $\mathrm{Ca}$ derived from the lines of neutral atoms. According to Zhao \& Gehren (2000, Mg I), Idiart \& Thevenin (2000, Mg I and Ca I), and Mashonkina et al. (2007a, Ca I), non-LTE abundance corrections may reach 0.2 dex depending on stellar parameters. For the Cohen et al. sample, they are expected to be similar to that calculated for our program turnoff stars BD $+3^{\circ} 740$ and BD-13 3442 , $\Delta_{\mathrm{NLTE}}=+0.04$ dex for $\mathrm{Mg}$ and $\Delta_{\mathrm{NLTE}}=+0.14$ dex for Ca. Based on our calculations for HD 122563, we expect that the LTE abundances of $\mathrm{Mg}$ and $\mathrm{Ca}$ in the cool giants of the Johnson (2002) and Cayrel et al. (2004) samples may be larger in non-LTE by approximately 0.2 dex. We note that accounting for departures from LTE leads to nearly identical overabundances of $\mathrm{Mg}$ and $\mathrm{Ca}$ relative to $\mathrm{Fe}$ in the turnoff stars of Cohen et al. and the cool giants of Cayrel et al. with the $\mathrm{Ca} / \mathrm{Mg}$ abundance ratio close to solar value. This makes the conclusions of the cited papers about a similarly constant ratio between the yields of iron and the $\alpha$ process elements $\mathrm{Mg}$ and $\mathrm{Ca}$ in the early Galaxy more solid.

Aluminium. As for neutral magnesium and calcium, non-LTE leads to larger abundances of $\mathrm{Al}$ derived from the resonance lines of Al I compared to those from LTE analysis. However, departures from LTE for Al I depend strongly on effective temperature and metallicity. As was shown above, the metal-deficient turnoff stars are affected most strongly by $\mathrm{Al}$ abundance corrections reaching +0.86 dex for $\mathrm{BD}-13^{\circ} 3442$. When non-LTE effects are neglected, Galactic abundance trends have a large scatter of data for the stars of close metallicity. E.g. for their sample of VMP stars $(-3.8 \leq[\mathrm{Fe} / \mathrm{H}] \leq-1.5)$, Barklem et al. (2005) find the scatter of the order of 0.5 dex for the $[\mathrm{Al} / \mathrm{Fe}]$ and $[\mathrm{Al} / \mathrm{Mg}] \mathrm{ra}-$ tios, while the scatters are less than the typical abundance errors for $[\mathrm{Mg} / \mathrm{Fe}]$ and $[\mathrm{Ca} / \mathrm{Fe}]$. The data of Fulbright $(2002)$ on $[\mathrm{Al} / \mathrm{Fe}]$ reveals even larger scatter up to $1 \mathrm{dex}$ at $[\mathrm{Fe} / \mathrm{H}]<-2$.

Some abundance analyses (e.g. Carretta et al. 2002; Cayrel et al. 2004; Cohen et al. 2004) carried out under the LTE assumption use the non-LTE abundance corrections published in Baumüller \& Gehren (1997). We note that Baumüller \& Gehren (1997) give $\Delta_{\text {NLTE }}$ for the small grid of model atmospheres, and individual corrections obtained by interpolating for given stellar parameters should be considered as a coarse treatment of the non-LTE effects. Nevertheless even a rough approach leads to a dramatical change of Galactic abundance trends. For the sample of stars in the metallicity range $-3.6 \leq[\mathrm{Fe} / \mathrm{H}] \leq-2.4$, Carretta et al. obtain the average LTE abundance $[\mathrm{Al} / \mathrm{Fe}]=-0.59 \pm 0.15$, while the $\mathrm{Al}$ abundance increases up to $[\mathrm{Al} / \mathrm{Fe}]=+0.10 \pm 0.22$ when the non-LTE corrections are applied. A different case is the Cayrel et al. study. They use an inappropriate correction for their sample of cool giants, $\Delta_{\mathrm{NLTE}}=+0.65$ dex that corresponds to the model with $T_{\text {eff }}=5500 \mathrm{~K}, \log g=3.5,[\mathrm{Fe} / \mathrm{H}]=-3$. Our calculations for $\mathrm{HD} 122563$ show that the $\mathrm{Al}$ abundance correction is much smaller, $\Delta_{\mathrm{NLTE}}=+0.04 \mathrm{dex}$. The non-LTE abundances of Al presented by Cayrel et al. in their Fig. 8 are therefore overestimated.

Alkalis, $\mathrm{Na}$ I and $\mathrm{KI}$. Contrary to $\mathrm{Mg}$, $\mathrm{Ca}$, and $\mathrm{Al}$, non-LTE leads to smaller abundances of sodium and potassium compared to those determined in LTE. As was shown by Baumüller et al. (1998) and Andrievsky et al. (2007) and supported in this paper, departures from LTE for Na I depend strongly on the surface gravity and the element abundance of a star. For the cool giant HD 122563, the non-LTE abundance correction of Na I $\lambda 5889$ / $\lambda 5895$ is a $0.15 \mathrm{dex} / 0.20 \mathrm{dex}$ more negative compared to that for the turnoff star BD-13 3442 of close metallicity and $\mathrm{Na}$ abundance. For our program turnoff stars, $\Delta_{\text {NLTE }}$ consists of $-0.47 \mathrm{dex} /-0.39 \mathrm{dex}$ for the atmospheric parameters that match HD 84937 and decreases in absolute value with decreasing metallicity, by approximately 0.2 dex for $\Delta[\mathrm{Fe} / \mathrm{H}]=-0.5$. As for NaI, the non-LTE effects for K I increase with decreasing surface gravity. For alkalis, a full treatment of non-LTE line formation is therefore required for each individual star. Andrievsky et al. (2007) show a clear advantage of such an approach compared to the use of a constant non-LTE abundance correction. They achieve the small star-to-star variation in the $[\mathrm{Na} / \mathrm{Fe}]$ and $[\mathrm{Na} / \mathrm{Mg}]$ ratios comparable with the measurement errors and consistent results for the samples of VMP dwarfs and unmixed giants.

Stellar abundance ratios between the odd- $Z$ and even- $Z$ elements such as $\mathrm{Na} / \mathrm{Mg}, \mathrm{Al} / \mathrm{Mg}$, and $\mathrm{K} / \mathrm{Ca}$ are important for 
testing current nucleosynthesis models. We emphasize that exactly these abundance ratios are most affected by departures from LTE. E.g. non-LTE leads to an increase of the $\mathrm{Al} / \mathrm{Mg}$ ratio in the program turnoff stars by 0.77 dex. In contrary, the $\mathrm{Na} / \mathrm{Mg}$ ratio decreases, on average, by 0.42 dex. It is worth noting that our non-LTE abundance ratios $\mathrm{Al} / \mathrm{Mg}$ and $\mathrm{Na} / \mathrm{Mg}$ agree well with the Galaxy chemical evolution calculations of Timmes et al. (1995) for the corresponding metallicity range. An exception is a too low value of $[\mathrm{Al} / \mathrm{Mg}]$ in $\mathrm{HD} 122563$. As was noted, this star reveals an overall deficiency of the elements requiring for their synthesis a large flux of neutrons.

Our data on potassium with the mean non-LTE ratio $[\mathrm{K} / \mathrm{Ca}]=-0.19 \pm 0.06$ favor the Galaxy chemical evolution calculations of Goswami \& Prantzos (2000). Similar conclusion was drawn by Shimansky et al. (2003) and Zhang et al. (2006b) based on non-LTE determinations of the potassium abundance in the mildly metal-poor $([\mathrm{Fe} / \mathrm{H}]>-1)$ stars.

Due to the strong influence of non-LTE effects in the atmospheres of metal-deficient stars LTE abundance analyses of $\mathrm{Na}, \mathrm{Al}$, and $\mathrm{K}$ are nearly useless for an investigation of Galactic chemical evolution.

Neutron-capture elements $\mathrm{Sr}, \mathrm{Ba}$, and Eu. The LTE abundance analyses (see e.g. Fulbright 2002; Carretta et al. 2002; Barklem et al. 2005) determine a steep decline of the $[\mathrm{Ba} / \mathrm{Fe}]$ abundance ratios at $[\mathrm{Fe} / \mathrm{H}]<-2$. Based on our non-LTE calculations we expect that the trend might be shallower in non-LTE. E.g. for the program turnoff stars, $[\mathrm{Ba} / \mathrm{Fe}]$ decreases from 0.00 at $[\mathrm{Fe} / \mathrm{H}]=-2.15$ down to -0.23 at $[\mathrm{Fe} / \mathrm{H}]=-2.66$ in non-LTE, while from -0.15 down to -0.73 in LTE.

As found in many studies (for a review of the literature under 2004; see Travaglio et al. 2004; and the later study of Barklem et al. 2005; they all computed under LTE), the abundance trends of $\mathrm{Sr} / \mathrm{Fe}, \mathrm{Ba} / \mathrm{Fe}, \mathrm{Eu} / \mathrm{Fe}$, and the ratios among neutron-capture elements have a large scatter at fixed metallicity that appears to increase with decreasing $[\mathrm{Fe} / \mathrm{H}]$. A part of the dispersions may be due to ignoring deviations from LTE in abundance determinations, because the non-LTE effects for the lines of Sr II and Ba II depend strongly on stellar parameters and the element abundance.

The measured scatter is usually interpreted as cosmic in origin, because iron and neutron-capture elements are produced in different sites. We suggest to plot the ratios among neutroncapture elements against the abundance of $\mathrm{Ba}$, but not the $\mathrm{Fe}$ abundance. In Fig. 7, we combine the $\mathrm{Sr} / \mathrm{Ba}$ abundance ratios from Barklem et al. (2005) with our data. Except for the few outliers, the scatter at fixed $\mathrm{Ba}$ abundance does not exceed 0.5 dex at $[\mathrm{Ba} / \mathrm{H}]>-3.5$ and approaches to $1 \mathrm{dex}$ at the lower $[\mathrm{Ba} / \mathrm{H}]$. This makes possible to see a growth of $\mathrm{Sr} / \mathrm{Ba}$ towards the lower $\mathrm{Ba}$ abundance. For comparison, the scatter in the run $[\mathrm{Sr} / \mathrm{Ba}]-[\mathrm{Fe} / \mathrm{H}]$ (see Fig. 22 from Barklem et al.) approaches to 1.5 dex at $[\mathrm{Fe} / \mathrm{H}]<-2.5$ and hides the correlations between the abundance ratios. According to the recent study of Mashonkina et al. (2007b), the $\mathrm{Zr} / \mathrm{Ba}$ and $\mathrm{Y} / \mathrm{Ba}$ abundance ratios in halo stars grow towards the lower $\mathrm{Ba}$ abundance. A clear abundance trend seen in Fig. 7 indicates an existence of the process that yielded in the early Galaxy strontium with little contribution to Ba. The present study supports the ideas of Truran et al. (2002) and observational findings of Aoki et al. (2005) and Honda et al. (2006) that the light and heavy neutron-capture elements in VMP stars come from distinct processes. Synthesis of the light neutroncapture elements remains a challenge to theorists.

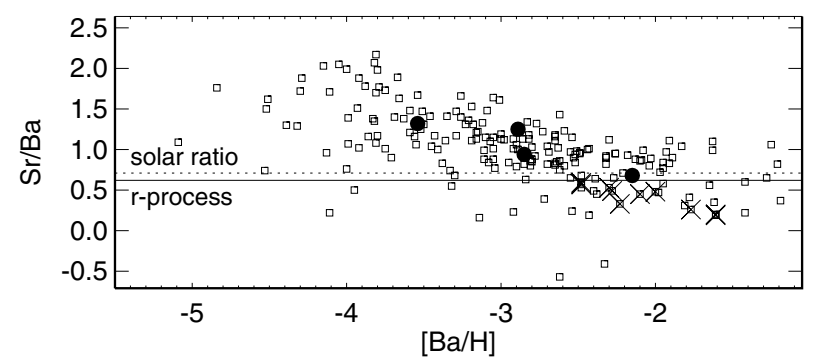

Fig. 7. A run of $\mathrm{Sr} / \mathrm{Ba}$ vs. $[\mathrm{Ba} / \mathrm{H}]$ for the stars of Barklem et al. (2005) sample (open squares) and our program stars (filled circles). Here, $\mathrm{Sr} / \mathrm{Ba}=\log \left(N_{\mathrm{Sr}} / N_{\mathrm{Ba}}\right)$ where $N_{X}$ are number densities. The $r$-process rich (r-II) stars are marked by crosses. The solar total abundance ratio $\mathrm{Sr} / \mathrm{Ba}=0.71$ obtained in this study is indicated by dotted line and the relative solar system $r$-process abundance $(\mathrm{Sr} / \mathrm{Ba})_{r}=0.62$ according to Arlandini et al. (1999) by solid line.

\section{The r/s-process controversy for HD 84937 and HD 122563}

\section{1. $[\mathrm{Eu} / \mathrm{Ba}]$ abundance ratios}

It is well known (among many observational studies see e.g. Spite \& Spite 1978; and Barklem et al. 2005) that in old metalpoor galactic stars europium is overabundant relative to $\mathrm{Ba}$. This is interpreted as a dominance of the $r$-process in production of heavy elements beyond the iron group in the early Galaxy. The solar system abundance ratio of $\mathrm{Eu}$ to $\mathrm{Ba}$ contributed by the $r$-process relative to the total abundances, $[\mathrm{Eu} / \mathrm{Ba}]_{r}$, equals 0.70 according to Arlandini et al. (1999). In several studies, Sneden et al. (1996, 2000), Cowan et al. (1999, 2002), and Hill et al. (2002) have presented arguments supporting constant relative $r$-process element abundances during the history of the Galaxy (at least, where $56 \leq Z<70$ ). In two stars, BD $+3^{\circ} 740$ and $\mathrm{BD}-13^{\circ} 3442$, the $\mathrm{Eu}$ abundance is not determined. Our computations for $[\mathrm{Eu} / \mathrm{Fe}]=0.5$ (which translates to $[\mathrm{Eu} / \mathrm{Ba}]=$ 0.7) predict the central depth of Eu II $\lambda 4129$ in these stars at a level of $0.5 \%$. Such a weak line cannot be measured in the spectra available to us. Thus, the Eu abundance in $\mathrm{BD}+3^{\circ} 740$ and $\mathrm{BD}-13^{\circ} 3442$ may be large enough to give a $[\mathrm{Eu} / \mathrm{Ba}] \mathrm{ra}-$ tio close to 0.7 . We obtain that HD 84937 reveals abundances close to a pure $r$-process production of heavy elements with $[\mathrm{Eu} / \mathrm{Ba}]=0.70$. For HD 122563, the corresponding abundance ratio is smaller, $[\mathrm{Eu} / \mathrm{Ba}]=0.50$, that suggests a contribution from the main $s$-process to observed barium.

\subsection{The fraction of the odd isotopes of $\mathrm{Ba}$}

A determination of the Ba even-to-odd isotope abundance ratio in stars becomes possible due to the significant hyperfine structure (HFS) affecting the Ba II resonance lines of the odd isotopes. In total, the resonance line $\lambda 4554$ has 15 components spread over $58 \mathrm{~m} \AA$. The larger the fraction of the odd isotopes, the stronger the HFS broadening of $\lambda 4554$ and the larger the energy absorbed in this line. The HFS broadening of the Ba II subordinate lines is small. The maximal effect on the derived abundance is found for $\lambda 6496$ and it does not exceed 0.01 dex for the Sun and is much smaller for our program metal-poor stars. We use the method described in detail in our previous study (Mashonkina \& Zhao 2006) and find the Ba even-to-odd isotope abundance ratio in a given star from the requirement that $\mathrm{Ba}$ abundances derived from the $\mathrm{Ba}$ II resonance line and the subordinate lines must be equal. 
For both stars, HD 84937 and HD 122563, the only subordinate line, Ba II $\lambda 6496$, is used to determine total Ba abundance. It is given in Table 4. The Ba II $\lambda 5853$ line cannot be extracted from noise in HD 84937 and is affected by a hot pixel in HD 122563. We do not use Ba II $\lambda 6141$ due to blending with a Fe I line.

Having fixed the $\mathrm{Ba}$ abundance of HD $84937([\mathrm{Ba} / \mathrm{Fe}]=0)$ and varying the even-to-odd isotope abundance ratio, we obtain a consistent element abundance from $\lambda 4554$ for a fraction of the odd isotopes $f_{\text {odd }}=0.43 \pm 0.14$. The uncertainty of the desired value is mainly caused by the uncertainty of the microturbulence velocity, which is estimated as $+0.2 \mathrm{~km} \mathrm{~s}^{-1}$. Recent $r$-process calculations of Kratz et al. (2007) predict $f_{\text {odd }}=0.44$. A close value, $f_{\text {odd }}=0.46$, is obtained from $r$-residuals in the stellar model of Arlandini et al. (1999). Thus, both the [Eu/Ba] abundance ratio and the even-to-odd $\mathrm{Ba}$ isotope abundance ratio suggest a pure $r$-process production of heavy neutron-capture elements in HD 84937.

For HD 122563, we find $f_{\text {odd }}=0.22 \pm 0.15$ with the uncertainty mainly caused by the uncertainties of $T_{\text {eff }}(120 \mathrm{~K})$ and $V_{\text {mic }}\left(0.1 \mathrm{~km} \mathrm{~s}^{-1}\right)$ (see Table 5). This fraction is close to the solar system value (0.18). Taking into account the $1 \sigma$ error, we estimate the $r$-process contribution to $\mathrm{Ba}$ in $\mathrm{HD} 122563$ to be no larger than $70 \%$ in agreement with the $[\mathrm{Eu} / \mathrm{Ba}]$ abundance ratio in this star. The only halo star with a measured fraction of the odd isotopes of $\mathrm{Ba}$ is HD 140283 (Lambert \& Allende Prieto 2002). Similarly to HD 122563 , it shows a lower value $f_{\text {odd }}=0.31$ compared with that of two other halo stars with a measured fraction of the odd isotopes of Ba, HD 84937 and HD $103095\left(f_{\text {odd }}=\right.$ 0.42 , Mashonkina \& Zhao 2006). We note that both HD 140283 and HD 122563 reveal lower neutron-capture element abundances compared to those of the stars of close metallicity and look as $r$-process-poor stars. For HD 140283, $[\mathrm{Ba} / \mathrm{Fe}]=-0.80$ according to Mashonkina et al. (2007b). Using the same stellar parameters $T_{\mathrm{eff}}=5810 \mathrm{~K}, \log g=3.68$, and $[\mathrm{Fe} / \mathrm{H}]=-2.43$, we estimate $[\mathrm{Eu} / \mathrm{Fe}]=-0.31$ based on the UVESPOP observed spectrum (Bagnulo et al. 2005) and non-LTE line formation. The obtained ratio $[\mathrm{Eu} / \mathrm{Ba}]=0.49$ is smaller than the value typical of the halo stars (e.g. $[\mathrm{Eu} / \mathrm{Ba}]=0.69$ deduced by McWilliam 1998 as the mean ratio for the sample of stars with $[\mathrm{Fe} / \mathrm{H}] \leq-2.4$ ) and smaller than the relative solar system $r$-process abundance $[\mathrm{Eu} / \mathrm{Ba}]_{r}=0.70$ (Arlandini et al. 1999).

\section{Conclusions}

In this paper, we determine for the first time stellar parameters $T_{\text {eff }}$ and $\log g$ of four VMP stars $(-2.15 \geq[\mathrm{Fe} / \mathrm{H}] \geq-2.66)$ based on non-LTE line formation. Effective temperature is derived from the Balmer $\mathrm{H}_{\alpha}$ and $\mathrm{H}_{\beta}$ line wing fits. For each star, non-LTE leads to a consistency of $T_{\text {eff }}$ from two lines and to a higher temperature compared to the LTE case, by up to $60 \mathrm{~K}$.

We find a clear advantage of the non-LTE approach in spectroscopic determination of surface gravity compared to the LTE case. For each of three stars with HIPPARcos parallax available, a surface gravity obtained from the non-LTE ionization balance between $\mathrm{Ca}$ I and $\mathrm{Ca}$ II agrees within the error bars with a trigonometric one. The $\mathrm{Ca} / \mathrm{Ca}$ II line intensity ratio involving Ca II $\lambda 8498$ is particularly sensitive to a variation of surface gravity. However, the wings of the $\mathrm{Ca}$ II resonance lines can also be used for spectroscopic determination of $\log g$ of VMP stars. We conclude that non-LTE calculations based on 1D model atmospheres provide reliable results for $T_{\text {eff }}$ and $\log g$ of VMP stars in the wide range of temperatures $\left(T_{\text {eff }}=4600 \mathrm{~K}\right.$ to $6400 \mathrm{~K})$ and gravities $(\log g=4.0$ down to 1.5$)$.
Due to strong influence of non-LTE effects in the atmospheres of VMP stars abundances of $\mathrm{Na}, \mathrm{Mg}, \mathrm{Al}, \mathrm{K}, \mathrm{Ca}, \mathrm{Sr}, \mathrm{Ba}$, and Eu cannot be determined in a reliable way assuming LTE. Departures from LTE depend strongly on stellar parameters such that a precise abundance analysis is only possible on the base of non-LTE calculations for each individual star.

We derive the fraction of the odd isotopes of $\mathrm{Ba}$ in two stars, HD 84937 and HD 122563, and, thus, increase the number of halo stars with a measured $f_{\text {odd }}$ to four. Two stars among four have low $f_{\text {odd }}$ : HD 122563 with $f_{\text {odd }}=0.22 \pm 0.15$ (this study) and HD 140283 with $f_{\text {odd }}=0.31$ (Lambert \& Allende Prieto 2002). Both stars reveal the lower Eu/Ba ratios compared to the relative solar system $r$-process abundances. These results suggest that the $s$-process has significantly contributed to the Ba abundance in these stars, or that the unknown process that produced high $\mathrm{Sr} / \mathrm{Ba}$ ratios in these stars yielded the low $f_{\text {odd }}$ value. Two remaining stars have the larger fraction of the odd isotopes of $\mathrm{Ba}: f_{\text {odd }}=0.43 \pm 0.14$ in $\mathrm{HD} 84937$ (this study) and $f_{\text {odd }}=0.42$ in HD 103095 (Mashonkina \& Zhao 2006). In both stars, the $\mathrm{Eu} / \mathrm{Ba}$ ratios are consistent with the relative solar system $r$-process abundances. We conclude therefore, that $f_{\text {odd }}$ found in these stars can serve as observational constraint on $r$ process models.

Acknowledgements. The authors thank Manuel Bautista for providing with the new collisional data on CaII and Keith Butler for his help. M.L. acknowledges with gratitude the National Astronomical Observatories of Chinese Academy of Science for warm hospitality during a productive stay in September of 2006. This research was supported by the Russian Foundation for Basic Research with grant 05-02-39005-GFEN-a, the Natural Science Foundation of China with grants NSFC 10433010, 10521001, and 10778612, the Deutsche Forschungsgemeinschaft with grant 436 RUS 17/13/07, the RF President with a grant on Leading Scientific Schools 784.2006.2, and the Presidium RAS Programme "Origin and evolution of stars and galaxies". We thank the anonymous referee for constructive suggestions and useful remarks.

\section{References}

Aldenius, M., Tanner, J. D., Johansson, S., et al. 2007, A\&A, 461, 767 Ali, A. W., \& Griem, H. R., 1966, Phys. Rev., 144, 366

Alonso, A., Arribas, S., \& Martínez-Roger, C. 1996, A\&AS, 117, 227 Alonso, A., Arribas, S., \& Martínez-Roger, C. 1999, A\&AS, 139, 335 Anders, E., \& Grevesse, N. 1989, Geoch., \& Cosmochim Acta, 53, 197 Andrievsky, S. M., Spite, M., Korotin, S. A., et al. 2007, A\&A, 464, 1081 Anstee, S. D., \& O'Mara, B. J. 1995, MNRAS, 276, 859 Aoki, W., Honda, S., Beers, T. C., et al., 2005, ApJ, 632, 611 Aoki, W., Frebel, A., Christlieb, N., et al. 2006, ApJ, 639, 897 Arlandini, C., Käppeler, F., Wisshak, K., \& et al., 1999, ApJ, 525, 886 Asplund, M. 2005, ARA\&A, 43, 481

Asplund, M., Grevesse, N., \& Sauval, A. J. 2005, ASP Conf. Ser., 336, 25 Asplund, M., Lambert, D. L., Nissen, P. E., et al. 2006, ApJ, 644, 229 Auer, L. H., \& Heasley, J. 1976, ApJ, 205, 165

Bagnulo, S., Jehin, E., Ledoux, C., et al. 2003, ESO Messenger, 114, 10 Barbuy, B., Meléndez, J., Spite, M., et al. 2003, ApJ, 588, 1072

Barklem, P. S. 2006, private communication

Barklem, P. S. 2007, A\&A, 466, 327

Barklem, P. S., \& Aspelund-Johansson, J. 2005, A\&A, 435, 373

Barklem, P. S., \& O’Mara, B. J. 1998, MNRAS, 300, 863

Barklem, P. S., \& Piskunov N. 2003, in Modelling of Stellar Atmospheres, ed. N. Piskunov, W. W. Weiss, \& D. F., Gray, Proc. IAU Symp., 210 Barklem, P. S., Piskunov, N., \& O’Mara, B. J. 2000, A\&A, 355, L5 (BPO) Barklem, P. S., Christlieb, N., Beers, T. C., et al., 2005, A\&A, 439, 129 Baumüller, D., \& Gehren, T. 1996, A\&A, 307, 961

Baumüller, D. \& Gehren, T. 1997, A\&A, 325, 1088

Baumüller, D., Butler, K., \& Gehren, T. 1998, A\&A, 338, 637

Belyakova, E. V., \& Mashonkina, L. I. 1997, Astron. Rep., 41, 530

Bethe, H. A., \& Salpeter, E. E. 1957, Quantum Mechanics of One- and TwoElectron Atoms (New York: Academic Press)

Butler, K. 2000, unpublished

Butler, K., \& Giddings, J. 1985, Newsletter on the analysis of astronomical spectra No. 9, University of London

Carretta, E., Gratton, R. G., Cohen, J. G., et al. 2002, AJ, 124, 481 
Cayrel, R., Depagne, E., Spite, M., et al. 2004, A\&A, 416, 1117 Christlieb, N., Bessel, M., Beers, T., et al. 2002, Nature, 419, 904 Christlieb, N., Gustafsson, B., Korn, A., et al. 2004, ApJ, 603, 708 Cohen, J. G., Christlieb, N., McWilliam, A., et al. 2004, ApJ, 612, 1107 Cowan, J. J., Pfeiffer, B., Kratz, K.-L., et al. 1999, ApJ, 521, 194 Cowan, J. J., Sneden, C., Burles, S., et al. 2002, ApJ, 572, 861 Drawin, H. W. 1968, Z. Physik, 211, 404

Frebel, A., Aoki, W., Christlieb, N., et al. 2005, Nature, 434, 871 Fuhrmann, K. 1998, A\&A, 330, 626

Fuhrmann, K., Axer, M., \& Gehren, T. 1993, A\&A, 271, 451 Fuhrmann, K., Pfeiffer, M., Frank, C., et al., 1997, A\&A, 323, 909 Fuhrmeister, B., Short, C. I., \& Hauschildt, P. H. 2006, A\&A, 452, 1083 Fulbright, J. P. 2000, AJ, 120, 1841

Fulbright, J. P. 2002, AJ, 123, 404

Gehren, T., Liang, Y. C., Shi, J. R., et al., 2004, A\&A, 413, 1045

Gehren, T., Shi, J. R., Zhang, H. W., et al., 2006, A\&A, 451, 1065 Goswami, A., \& Prantzos, N., 2000, A\&A, 359, 191

Gratton, R. G., Carretta, E., Claudi, R., et al. 2003, A\&A 404, 187 Grupp, F., 2003, A\&A, 412, 897

Hill, V., Plez, B., Cayrel, R., et al. 2002, A\&A, 387, 560

Honda, S., Aoki, W., Ishimaru, Y., et al. 2006, ApJ, 643, 1180

Idiart, T. P., \& Thevenin, F. 2000, ApJ, 541, 207

Ivans, I. I., Sneden, C., James, C. R., et al. 2003, ApJ, 592, 906

Johnson, J. A. 2002, ApJS, 139, 219

Johnson, L. C. 1972, ApJ, 174, 227

Kamp, I., Korotin, S., Mashonkina, L., et al. 2003, in Modelling of Stellar Atmospheres. Proc. IAU Symp. 210, Uppsala, 17-21 June 2002, ed. N. Piskunov, W. W. Weiss, \& D. F. Gray, 323

Korn, A. 2002, in Scientific Drivers for ESO Future VLT/VLTI Instrumentation, ed. J. Bergeron, \& G. Monnet, (Berlin: Springer-Verlag), 199

Korn, A., Shi, J., \& Gehren, T. 2003 A\&A, 407, 691

Kratz, K.-L., Farouqi, Kh., Pfeiffer, B., et al. 2007, ApJ, 662, 39

Kupka, F., Piskunov, N., Ryabchikova, T. A., et al. 1999, A\&AS, 138, 119

Kurucz, R. L. 1992, CD-ROM No. 18; CD-ROM No. 23

Kurucz, R. L., Furenlid, I., Brault, J., \& Testerman, L. 1984, Solar Flux Atlas from 296 to $1300 \mathrm{~nm}$ Nat. Solar Obs., Sunspot, New Mexico

Lambert, D. L., \& Allende Prieto, C. 2002, MNRAS, 335, 325

Landstreet, J. D. 1998, A\&A, 338, 1041

Lawler, J. E., Wickliffe, M. E., Den Hartog, E. A., \& Sneden, C. 2001, ApJ, 563, 1075

Mashonkina, L. I. 2000, Astron. Rep. 44, 558

Mashonkina, L. I., \& Gehren, T. 2000, A\&A, 364, 249

Mashonkina, L. I., \& Gehren, T. 2001, A\&A, 376, 232

Mashonkina, L., \& Vinogradova, A. 2007, in preparation

Mashonkina, L. I., \& Zhao, G. 2006, A\&A, 456, 313

Mashonkina, L. I., Gehren, T., \& Bikmaev, I. F. 1999, A\&A, 343, 519

Mashonkina, L. I., Korn, A. J., \& Przybilla, N. 2007a, A\&A, 461, 261

Mashonkina, L., Vinogradova, A., Ptitsyn, D., et al. 2007b, Astron. Rep., 84, 997

McWilliam, A. 1998, AJ, 115, 1640

McWilliam, A., Preston, G. W., Sneden, C., \& Searle, L. 1995, AJ, 109, 2757

Meléndez, J., \& Ramírez, I. 2004, ApJ, 615, L33

Meléndez, M., Bautista, M. A., \& Badnell, N. R. 2007, A\&A, 469, 1203
Mihalas, D. 1978, Stellar Atmospheres, 2nd. edn. (San Francisco: Freeman)

Mishenina, T. V., Kovtyukh, V. V., Korotin, S. A., \& Soubiran, C. 2003, ARep 47,422

Moity, J. 1983, A\&AS, 52, 37

Moore, C. E. 1972, A Multiplet Table of Astrophysical Interest. NSRDS-NBS 40

Noguchi, K., Aoki, W., Kawanomoto, S., et al. 2002, PASJ, 54, 855

Nörtershäuser, W., Blaum, K., Icker, K., et al. 1998, Eur. Phys. J., D2, 33

Perryman, M. A.C., de Boer, K. S., Gilmore, G., et al. 2001, A\&A, 369, 339

Przybilla, N., \& Butler, K. 2004, ApJ, 609, 1181

Przybilla, N., \& Butler, K. 2004, ApJ, 610, L61

Raassen, A. J. J., \& Uylings, P. H.M. 1998, A\&A, 340, 300

Reader, J., Corliss, C. H., Wiese, W. L., \& Martin, G. A. 1980, Wavelengths and Transition Probabilities for Atoms and Atomic Ions. Part II. NSRDS - NBS 68, Washington, D. C.

Rybicki, G. B., \& Hummer, D. G. 1991, A\&A, 245, 171

Rybicki, G. B., \& Hummer, D. G. 1992, A\&A, 262, 209

Sakhibullin, N. A. 1983, Trudi Kazan gor. obs., 48, 9

Seaton, M. J., Mihalas, D., \& Pradhan, A. K. 1994, MNRAS, 266, 805

Shi, J. R., Gehren, T., \& Zhao, G. 2004, A\&A, 423, 683

Shimanskaya, N. N., \& Mashonkina, L. I. 2001, Astr. Rep, 45, 100

Shimansky, V. V., Bikmaev, I. F., Galeev, A. I., et al., 2003, Astr. Rep, 47, 750

Short, C. I., \& Hauschildt, P. H. 2005, ApJ, 618, 926

Simmerer, J., Sneden, C., Cowan, J. J., et al. 2004, ApJ, 617, 1091

Smith, G. 1981, A\&A, 103, 351

Smith, G., \& O'Neil, J. A. 1975, A\&A, 38, 1

Smith, G., \& Raggett, D. St. J. 1981, J. Phys., B14, 4015

Sneden, C., McWilliam, A., Preston, G. W., et al. 1996, ApJ, 467, 819

Sneden, C., Johnson, J., Kraft, R. P., et al. 2000, ApJ, 536, L85

Spergel, D. N., Verde, L., \& Peiris, H. V. 2003, ApJS, 148, 175

Spite, M., Cayrel, R., Hill, V., et al. 2006, A\&A, 455, 291

Spite, M., \& Spite, F. 1978, A\&A, 67, 23

Steenbock, W., \& Holweger, H. 1984, A\&A, 130, 319

Takeda, Y., Zhao, G., Chen, Y., et al., 2002, PASJ, 54, 275

Theodosiou, C. E. 1989, Phys. Rev., A39, 4880

Thevenin, F., \& Idiart, T. P. 1999, ApJ, 521, 753

Timmes, F. X., Woosley S. E., \& Weaver, T. A. 1995, ApJS, 98, 617

Travaglio, C., Gallino, R., Arnone, E., et al. 2004, ApJ, 601, 864

Truran, J. W., Cowan, J. J., Pilachowski, C. A., \& Sneden, C. 2002, PASP, 114, 1293

VandenBerg, D. A., Swenson, F. J., Rogers, F. J., et al. 2000, ApJ, 532, 430

Vidal, C. R., Cooper, J., \& Smith, E. W., 1970, JQSRT, 10, 1011

Vidal, C. R., Cooper, J., \& Smith, E. W., 1973, ApJS 25, 37

Wiese, W. L., Smith, M. W., \& Glennon, B. M. 1966, Atomic transition probabilities. Vol.: Hydrogen through Neon, A critical data compilation, NSRDSNBS 4, Washington, D. C.: US Department of Commerce, National Bureau of Standards

Zhang, H. W., Butler, K., Gehren, T., et al. 2006a, A\&A, 453, 723

Zhang, H. W., Gehren, T., Butler, K., et al. 2006b, A\&A, 457, 645

Zhao, G., \& Gehren, T. 2000, A\&A, 362, 1077

Zhao, G., Butler, K., \& Gehren, T. 1998, A\&A, 333, 219 